\title{
Evolution and Stability of Two-Dimensional Anelastic Internal Gravity Wave Packets
}

\author{
Alain D. Gervais AND GORdon E. Swaters \\ Department of Mathematical and Statistical Sciences, University of Alberta, Edmonton, Alberta, Canada \\ TON S. VAN DEN BREMER \\ Department of Engineering Science, University of Oxford, Oxford, United Kingdom
}

BRUCE R. SUTHERLAND

Departments of Physics, and Department of Earth and Atmospheric Sciences, University of Alberta, Edmonton, Alberta, Canada

(Manuscript received 19 December 2017, in final form 8 August 2018)

\begin{abstract}
The weakly nonlinear evolution, stability, and overturning of horizontally and vertically localized internal gravity wave packets is examined for a nonrotating, anelastic atmosphere that is stationary in the absence of waves. The weakly nonlinear evolution is examined through the derivation of their wave-induced mean flow, which is used to formulate a nonlinear Schrödinger equation. The induced flow is manifest as a long, hydrostatic, bow wake-like disturbance, whose flow direction transitions from positive on the leading flank of the wave packet to negative on the trailing flank of the wave packet. As such, two-dimensional wave packets are always modulationally unstable. This instability results in enhanced amplitude growth confined to either the leading or trailing flank. Hence, when combined with anelastic growth predicted by linear theory, we anticipate two-dimensional waves will overturn either somewhat below or just above the heights predicted by linear theory. Numerical solutions of the Schrödinger equation are compared with the results of fully nonlinear simulations to establish the validity of the weakly nonlinear theory. Actual wave overturning heights are determined quantitatively from a range of fully nonlinear simulations.
\end{abstract}

\section{Introduction}

Internal gravity waves propagate vertically within continuously stratified fluids. In the atmosphere, upwardpropagating waves experience exponential amplitude growth as a consequence of momentum conservation, owing to the approximately exponentially decreasing atmospheric density (Eliassen and Palm 1961). Once their amplitude becomes sufficiently large they overturn and eventually break, thus irreversibly depositing momentum to the background winds (McFarlane 1987). Anelastic growth, predicted by linear theory, combined with critical level interactions, was considered by Lindzen (1981) to be the most important means by which wave breaking and momentum deposition ultimately occur. These effects were parameterized in general circulation models using so-called gravity wave

Corresponding author: Bruce Sutherland, bruce.sutherland@ ualberta.ca drag schemes to estimate the vertical levels at which subgrid-scale internal gravity waves would deposit their momentum (Palmer et al. 1986; McFarlane 1987; Scinocca and McFarlane 2000). Of course, before reaching overturning amplitudes, the waves may nonetheless be of sufficiently large amplitude that linear theory incorrectly predicts their evolution. Indeed, in their study of horizontally periodic, vertically localized anelastic wave packets, Dosser and Sutherland (2011, hereafter DS11) found in particular that the modulational stability of hydrostatic waves retarded the growth of waves to such a degree that overturning did not occur until many density scale heights above the overturning level predicted by linear theory. As a nontrivial extension of DS11, the effect of weakly and fully nonlinear dynamics on twodimensional anelastic wave packets is investigated here.

The primary mechanism governing the weakly nonlinear evolution of one-dimensional (horizontally periodic, vertically localized) finite-amplitude wave packets is the interaction between the waves and their induced 
mean flow, an order-amplitude-squared forcing that acts to modify the structure of the wave packet as it propagates (Sutherland 2006a). An explicit expression for the mean flow induced by one-dimensional Boussinesq wave packets has been known since Acheson (1976). This flow is horizontally uniform and unidirectional. A corresponding expression for the flow induced by onedimensional wave packets in an anelastic gas was derived by Scinocca and Shepherd (1992) and DS11. The flow induced by two-dimensional (horizontally and vertically localized) Boussinesq wave packets is qualitatively different (Bretherton 1969). Rather than being unidirectional, the induced flow resembles a bow wake extending far horizontally and below the wave packet. Crucially, the flow changes sign from positive to negative from the leading to the trailing edge of the wave packet. Here we show these qualitative features extend to two-dimensional anelastic wave packets.

The weakly nonlinear evolution of internal gravity wave packets, through its dependence on interactions between the waves and their induced mean flow, is described by the nonlinear Schrödinger equation. Such an equation has been derived for one-dimensional wave packets in a uniformly stratified, nonrotating Boussinesq fluid with no background flow (Akylas and Tabaei 2005; Tabaei and Akylas 2007; Sutherland 2006b). The corresponding nonlinear Schrödinger equation for onedimensional wave packets in an anelastic gas was derived by DS11. The effect of the wave-induced mean flow is conceptually identical in both situations: the induced flow acts through the weakly nonlinear dispersion relation to Doppler-shift the waves, which in turn modifies the vertical group speed. Depending on the initial frequency of the waves, this results either in amplitude decay (modulational stability) or in amplitude growth (instability). Consequently, DS11 found that modulationally unstable waves (with frequency higher than that of waves with the fastest vertical group velocity) overturned at a lower height than that predicted by linear theory, and they found that hydrostatic waves, being modulationally stable, could travel without breaking multiple density scale heights above the overturning height predicted by linear theory.

A nonlinear Schrödinger equation for effectively twodimensional Boussinesq wave packets was first derived by Shrira (1981). However, the nondimensionalization used in that study resulted in a priori assumptions about the relative magnitude of the nonlinear advection terms in the governing equations. Akylas and Tabaei (2005) and Tabaei and Akylas (2007) separately derived a (weakly) nonlinear evolution equation for two- and threedimensional Boussinesq wave packets. Here, we use the theory for quasi-monochromatic wave packets to derive the nonlinear Schrödinger equation for two-dimensional anelastic wave packets. Its range of validity is confirmed by comparison with the results of fully nonlinear simulations. Those simulations are also used to assess wave overturning, yielding qualitatively different results than those of DS11 for one-dimensional wave packets.

The wave-induced mean flow and the weakly nonlinear evolution equation for two-dimensional anelastic wave packets are derived in section 2. The numerical methods used to solve the weakly and fully nonlinear equations are described in section 3 . The results of the weakly nonlinear simulations are compared with the results of DS11 and with the results of the fully nonlinear simulations in section 4. Finally, in section 5 we discuss the results with particular consideration of topographically excited waves.

\section{Derivation of the weakly nonlinear equations}

Here we derive equations for the mean flow induced by a two-dimensional anelastic internal gravity wave packet and for the nonlinear Schrödinger equation that models its moderately large-amplitude evolution. The model atmosphere is assumed to be uniformly stratified and stationary in the absence of waves. The procedure to find the waveinduced mean flow follows that of van den Bremer and Sutherland (2014), who found the induced flow for twodimensional Boussinesq waves (see also Bretherton 1969; Tabaei and Akylas 2007; van den Bremer and Sutherland 2018). We neglect Coriolis effects and arbitrarily suppose the wave packets propagate in the $x-z$ plane. In the derivations for both the wave-induced mean flow and the nonlinear Schrödinger equation, our starting point is the equations of motion for an ideal, inviscid, anelastic gas (Ogura and Phillips 1962; Lipps and Hemler 1982):

$$
\frac{D \mathbf{u}}{D t}=-\nabla \frac{p}{\bar{\rho}}+\frac{g}{\bar{\theta}} \theta \hat{\mathbf{e}}_{z} ; \quad \frac{D \theta}{D t}=-w \frac{d \bar{\theta}}{d z} ; \quad \nabla \cdot(\bar{\rho} \mathbf{u})=0,
$$

where $D / D t=\partial_{t}+\mathbf{u} \cdot \nabla$ is the material derivative and $\hat{\mathbf{e}}_{z}$ is the standard unit basis vector in the $z$ direction. The velocity field is given by $\mathbf{u}=(u, w)$. In the internal energy Eq. (1b), $\theta$ and $\bar{\theta}$ are the fluctuation and background potential temperature fields, respectively. The background potential temperature is given generally by

$$
\bar{\theta}=\bar{T}\left(\bar{p} / p_{0}\right)^{-\kappa},
$$

where $\bar{T}$ is the background temperature, $\bar{p}$ and $p_{0}$ are the background and reference pressures, respectively, and $\kappa \approx 2 / 7$. Assuming an isothermal atmosphere, $\bar{T}=T_{0}=\theta_{0}$, and the background density and pressure decrease exponentially with height with $e$-folding depth $H_{\rho}=$ $-\bar{\rho}(d \bar{\rho} / d z)^{-1}=R T_{0} / g$, in which the background density, $\bar{\rho}$, is explicitly given by 


$$
\bar{\rho}=\rho_{0} e^{-z / H_{\rho}} .
$$

The corresponding background potential temperature is

$$
\bar{\theta}=\theta_{0} e^{z / H_{\theta}},
$$

where $H_{\theta}=H_{\rho} / \kappa$ is the potential temperature scale height. Finally, the squared buoyancy frequency is

$$
N^{2}=\frac{g}{\bar{\theta}} \frac{d \bar{\theta}}{d z}=\frac{g}{H_{\theta}}
$$

which is constant in our assumed isothermal atmosphere.

\section{a. Wave-induced mean flow}

The form of the wave-induced mean flow applicable to horizontally periodic, spanwise uniform (one-dimensional) internal gravity wave packets has been derived using the principle of wave action by Acheson (1976), and by Scinocca and Shepherd (1992) using Hamiltonian fluid dynamics. Alternatively, it was derived from momentum conservation of quasi-monochromatic wave packets (Sutherland 2010) to be

$$
U_{1 \mathrm{D}}=u_{\mathrm{DF}}=\frac{\langle u w\rangle}{c_{g_{z}}}=\frac{1}{2} N|\mathbf{k}||A|^{2} .
$$

Here $\langle\cdot\rangle$ denotes the horizontal average, $c_{g_{z}}$ is the vertical group velocity, $\mathbf{k}=(k, m)$ is the wavenumber vector, and $A$ is the vertical displacement amplitude envelope, which is a function of $z$ and $t$ for one-dimensional wave packets. The subscript "DF" in (6) indicates that the flow results from the divergence of the momentum flux per unit mass. For a one-dimensional wave packet, this is a horizontally uniform, unidirectional flow. As such, $u_{\mathrm{DF}}$ is itself nondivergent, and so the total induced flow $U_{1 \mathrm{D}}$ exactly equals $u_{\mathrm{DF}}$. For a two-dimensional spanwise-uniform wave packet, the amplitude varies in $x$ as well as in $z$ and $t$. Therefore, $u_{\mathrm{DF}}$ in (6) is a divergent flow and pressure gradients are established to ensure (1) is satisfied. In a Boussinesq fluid, the resulting total induced flow is manifest as a horizontally long wave resembling a bow wake, with the flow above the vertical center of the wave packet being oppositely signed to the flow below the center (Bretherton 1969; Akylas and Tabaei 2005; Tabaei and Akylas 2007; van den Bremer and Sutherland 2014).
Following the approach of van den Bremer and Sutherland (2014) for two-dimensional Boussinesq quasi-monochromatic wave packets, here we extend their results to determine the flow induced by twodimensional wave packets in an anelastic gas. Equation (1b) is recast in terms of the vertical displacement, given by $\xi=-\theta / \bar{\theta}^{\prime}$, where the prime denotes differentiation with respect to $z$, so that

$$
\frac{D \xi}{D t}=w
$$

We eliminate the pressure terms in (1a) by taking the curl of the momentum equations which yields an equation for the evolution of spanwise vorticity,

$$
\frac{D \zeta}{D t}=-\frac{1}{H_{\rho}} \zeta w+N^{2} \frac{\partial \xi}{\partial x} .
$$

The anelastic condition (1c) allows us to write the velocity fields as density-normalized derivatives of the mass streamfunction $\Psi$, defined implicitly by the relations

$$
u=-\frac{1}{\bar{\rho}} \frac{\partial \Psi}{\partial z} ; \quad w=\frac{1}{\bar{\rho}} \frac{\partial \Psi}{\partial x} .
$$

Consequently, the vorticity $\zeta \equiv \partial_{z} u-\partial_{x} w$ is related to the mass streamfunction via

$$
\zeta=-\frac{1}{\bar{\rho}}\left(\nabla^{2} \Psi+\frac{1}{H_{\rho}} \frac{\partial \Psi}{\partial z}\right)
$$

Substituting (9) and (10) into (8) yields an expression for the nonlinear evolution of the mass streamfunction,

$$
\begin{aligned}
\frac{D}{D t} & {\left[\frac{1}{\bar{\rho}}\left(\nabla^{2} \Psi+\frac{1}{H_{\rho}} \frac{\partial \Psi}{\partial z}\right)\right] } \\
& =-N^{2} \frac{\partial \xi}{\partial x}-\frac{1}{H_{\rho} \bar{\rho}^{2}} \frac{\partial \Psi}{\partial x}\left(\nabla^{2} \Psi+\frac{1}{H_{\rho}} \frac{\partial \Psi}{\partial z}\right) .
\end{aligned}
$$

Combining the partial time derivative of (11) with $N^{2}$ times the partial $x$ derivative of (7) gives an equation for the mass streamfunction written as a linear operator $L$ acting on $\Psi$ on the left-hand side and the densityscaled divergence of a nonlinear vector $\mathbf{F}$ on the righthand side,

$$
\underbrace{\left[\partial_{t t}\left(\nabla^{2}+\frac{1}{H_{\rho}} \partial_{z}\right)+N^{2} \partial_{x x}\right]}_{\equiv L} \Psi=\bar{\rho} \nabla \cdot \underbrace{\left[\partial_{t}(\mathbf{u} \zeta)+N^{2} \partial_{x}(\mathbf{u} \xi)-\frac{N^{2}}{H_{\rho}} w \xi \hat{\mathbf{e}}_{x}\right]}_{\equiv \mathbf{F}} .
$$


TABLE 1. Expressions for the various fields at $O\left(\alpha \epsilon^{0}\right)$ and $O(\alpha \epsilon)$ in terms of the amplitude envelope function $A$ of the vertical displacement field. In each expression, subscripts on $A$ denote partial derivatives with respect to the slow-scale variables $X$ and $Z, \varphi \equiv k x+m z-\omega t$, and $K^{2} \equiv k^{2}+m^{2}+1 / 4 H_{\rho}^{2}$. By convention, the actual fields are taken to be the real parts of the above expressions.

\begin{tabular}{|c|c|c|}
\hline Field & $O\left(\alpha \varepsilon^{0}\right)$ & $O(\alpha \varepsilon)$ \\
\hline Vertical displacement & $\xi_{0}^{(1)}=A e^{i \varphi+z / 2 H_{\rho}}$ & $\xi_{1}^{(1)}=0$ \\
\hline Mass streamfunction & $\Psi_{0}^{(1)}=-\rho_{0} \frac{\omega}{k} A e^{i \varphi-z / 2 H_{\rho}}$ & $\Psi_{1}^{(1)}=-i \rho_{0} \frac{N}{K^{3}}\left(k A_{X}+m A_{Z}\right) e^{i \varphi-z / 2 H_{\rho}}$ \\
\hline Horizontal velocity & $u_{0}^{(1)}=\frac{\omega}{k}\left(i m-\frac{1}{2 H_{\rho}}\right) A e^{i \varphi+z / 2 H_{\rho}}$ & $u_{1}^{(1)}=\frac{N}{K^{3}}\left[i k\left(i m-\frac{1}{2 H_{\rho}}\right) A_{X}+\left(K^{2}-m^{2}-\frac{i m}{2 H_{\rho}}\right) A_{Z}\right] e^{i \varphi+z / 2 H_{\rho}}$ \\
\hline Vertical velocity & $w_{0}^{(1)}=-i \omega A e^{i \varphi+z / 2 H_{\rho}}$ & $w_{1}^{(1)}=\frac{N}{K^{3}}\left[\left(k^{2}-K^{2}\right) A_{X}+k m A_{Z}\right] e^{i \varphi+z / 2 H_{\rho}}$ \\
\hline Vorticity & $\zeta_{0}^{(1)}=-N K A e^{i \varphi+z / 2 H_{\rho}}$ & $\zeta_{1}^{(1)}=i \frac{N}{K}\left(k A_{X}+m A_{Z}\right) e^{i \varphi+z / 2 H_{\rho}}$ \\
\hline
\end{tabular}

Together, (7) and (12) form the set of coupled governing equations for $\xi$ and $\Psi$, which is closed by inclusion of the relations (9) and (10).

We seek approximate solutions of (12) corresponding to horizontally and vertically localized quasi-monochromatic wave packets, whose vertical displacement field is given by

$$
\xi_{0}^{(1)}=A_{0} \mathcal{A}(x, z, t) e^{i(k x+m z-\omega t)+z / 2 H_{\rho}},
$$

in which it is understood that the actual displacement is the real part of the right-hand-side expression. Here the superscript and subscript on $\xi$ denote the field's order in the perturbation parameters $\alpha$ and $\varepsilon$, respectively, in which $\alpha=A_{0} k$ is a nondimensional measure of the wave packet's maximum vertical displacement amplitude $A_{0}$ and $\varepsilon=1 /\left(k \sigma_{z}\right)$ is a nondimensional measure of the inverse vertical extent $\sigma_{z}$ of the wave packet. For simplicity, we suppose the scales of horizontal and vertical variations of the amplitude envelope are comparable. Thus, $x$ and $z$ derivatives of $\mathcal{A}$ are both of order $\varepsilon$.

The usual polarization relations for anelastic internal waves are found by extracting terms of $O\left(\alpha \varepsilon^{0}\right)$ in (1) and relating these fields to $\xi_{0}^{(1)}$ in (13). These results are given in the second column of Table 1. Separately extracting terms of $O(\alpha \varepsilon)$, which have exactly one spatial derivative of $\mathcal{A}$, we find the leading-order corrections to these fields for wave packets. These are given in the third column of Table 1.

Substituting the terms of $O\left(\alpha \varepsilon^{0}\right)$ into the right-hand side of (12) and extracting the slowly varying components of the products $\mathbf{u}_{0}^{(1)} \boldsymbol{\zeta}_{0}^{(1)}$ and $\mathbf{u}_{0}^{(1)} \xi_{0}^{(1)}$, we find $(\nabla \cdot \mathbf{F})_{2}^{(2)}=0$ at this order. In particular, we neglect the superharmonic terms, that is, those terms proportional to $e^{ \pm 2 i(k x+m z-\omega t)}$ (e.g., Grimshaw 1981; Shrira 1981; Tabaei and Akylas 2007; Achatz et al. 2010). That $(\nabla \cdot \mathbf{F})_{2}^{(2)}=0$ is in accordance with the well-known fact that plane periodic internal gravity waves exactly solve the fully nonlinear equations of motion. However, including terms of $O(\alpha \varepsilon)$ on the right-hand side of (12), we find a nontrivial expression for $\nabla \cdot \mathbf{F}$ at $O\left(\alpha^{2} \varepsilon^{3}\right)$ (see appendix A). For a twodimensional Boussinesq wave packet, Bretherton (1969) and van den Bremer and Sutherland (2014) replaced the $\tilde{x}$-dependent forcing in $(\nabla \cdot \mathbf{F})_{3}^{(2)}$ by a Dirac-delta function at the center of the wave packet of equivalent strength by integrating with respect to $\tilde{x}$ between $-\infty$ and $\infty$. Equivalently, but without the intermediate step of introducing a Dirac-delta function, we ignore $\tilde{x}$ derivatives to obtain

$$
\bar{\rho}(\nabla \cdot \mathbf{F})_{3}^{(2)} \approx-\rho_{0} \frac{1}{2} \frac{N^{3} k^{2} m^{2}}{K^{5}} A_{0}^{2} \partial_{\tilde{z} \tilde{z} \tilde{z}}|\mathcal{A}(\tilde{x}, \tilde{z})|^{2},
$$

in which $(\tilde{x}, \tilde{z})=\left(x-c_{g_{x}} t, z-c_{g_{z}} t\right)$ are coordinates translating at the group velocity of the wave packet and $K=\left[k^{2}+m^{2}+1 /\left(4 H_{\rho}\right)^{2}\right]^{1 / 2}$.

The expression (14) acts as an order-amplitude-squared forcing on the right-hand side of (12) that forces an order-amplitude-squared flow through the induced mass streamfunction $\Psi^{(2)}$. Casting the linear operator $L$ in (12) in terms of $\tilde{x}$ and $\tilde{z}$, and using the anticipated hydrostatic response so that $\partial_{t t} \sim c_{g_{z}}^{2} \partial_{\tilde{z} \tilde{z}}$ and $\partial_{\tilde{x} \tilde{x}}+\partial_{\tilde{z} \tilde{z}} \sim \partial_{\tilde{z} \tilde{z}}$ (Bretherton 1969; van den Bremer and Sutherland 2014), (12) becomes

$$
\begin{aligned}
& \left(c_{g_{z}}^{2} \partial_{\tilde{z} \tilde{z} \tilde{z} \tilde{z}}+\frac{1}{H_{\rho}} \partial_{\tilde{z} \tilde{z} \tilde{z}}+N^{2} \partial_{\tilde{x} \tilde{x}}\right) \Psi^{(2)} \\
& =-\frac{1}{2} \rho_{0} \frac{N^{3} k^{2} m^{2}}{K^{5}} A_{0}^{2} \partial_{\tilde{z} \tilde{z} \tilde{z}}|\mathcal{A}(\tilde{x}, \tilde{z})|^{2} .
\end{aligned}
$$

We solve for $\Psi^{(2)}$ by taking the double Fourier transform in $\tilde{x}$ and $\tilde{z}$ of the above expression, defining the Fourier transform as $\hat{A}(\kappa, \mu) \equiv(2 \pi)^{-2} \int_{\mathbb{R}^{2}} A(\tilde{x}, \tilde{z}) e^{-i(\kappa \tilde{x}+\mu \tilde{z})} d \tilde{x} d \tilde{z}$. In Fourier space the mass streamfunction is

$$
\widehat{\Psi^{(2)}}(\kappa, \mu)=\frac{i}{2} \rho_{0} \frac{N^{3} k^{2} m^{2}}{K^{5}} A_{0}^{2} \frac{\mu^{3} \mid \widehat{\left.\mathcal{A}\right|^{2}}}{c_{g_{z}}^{2} \mu^{4}-i \frac{1}{H_{\rho}} c_{g_{z}}^{2} \mu^{3}-N^{2} \kappa^{2}} .
$$

The real space solution of $\Psi^{(2)}$ is found by double inverse transforming the above expression, the result of which is 


$$
\Psi^{(2)}(\tilde{x}, \tilde{z})=\frac{i}{2} \rho_{0} \frac{N k^{2} m^{2}}{K^{5}} A_{0}^{2} \int_{\mathbb{R}^{2}} \frac{\mu^{3} \mid \widehat{\left.\mathcal{A}\right|^{2}} e^{i(\kappa \tilde{x}+\mu \tilde{z})}}{\frac{c_{z}^{2}}{N^{2}}\left(\mu^{4}-i \frac{1}{H_{\rho}} \mu^{3}\right)-\kappa^{2}} d \kappa d \mu
$$

The integral with respect to $\kappa$ can be determined explicitly, as was done for Boussinesq wave packets (the limit as $\left.H_{\rho} \rightarrow \infty\right)$ by van den Bremer and Sutherland (2014) (see also Bretherton 1969). For anelastic waves this task is nontrivial due to the presence of complex singularities in the integrand. Integration may be performed using residue theory, choosing integration contours to ensure causality as described in appendix B. In practice, however, it is numerically more convenient to compute the wave-induced momentum in Fourier space via (9a) and (16), so that

$$
\begin{aligned}
\widehat{\rho} u^{(2)}=-i \mu \widehat{\Psi^{(2)}}= & \frac{1}{2} \rho_{0} \frac{N k^{2} m^{2}}{K^{5}} A_{0}^{2} \frac{\mu^{4} \mid \widehat{\left.\mathcal{A}\right|^{2}}}{2 C(\mu)} \\
& \times\left[\frac{1}{\kappa+C(\mu)}-\frac{1}{\kappa-C(\mu)}\right] .
\end{aligned}
$$

Here,

$$
\begin{aligned}
C(\mu) & \equiv \frac{\left|c_{g_{z}}\right|}{N}\left(\mu^{4}-i \frac{1}{H_{\rho}} \mu^{3}\right)^{1 / 2} \\
& =\frac{\left|c_{g_{z}}\right|}{N}\left(\mu^{8}+\frac{1}{H_{\rho}^{2}} \mu^{6}\right)^{1 / 4}(\cos \Phi+i \sin \Phi) \\
& =C_{R}+i C_{I},
\end{aligned}
$$

where $\Phi= \pm(1 / 2) \tan ^{-1}\left(1 / H_{\rho}|\mu|\right)$, and the subscripts $R$ and $I$ denote the real and imaginary parts, respectively, of the function $C(\mu)$. To capture induced long waves that propagate outward with respect to the translating wave packet, when evaluating $C(\mu)$ one must choose the appropriate branch cut (Bretherton 1969). This is achieved here by choosing the plus-or-minus sign of $\Phi$ to be equal to $\operatorname{sgn}(\mu)$. The wave-induced momentum in real space is found by inverse transforming (18), that is,

$$
\bar{\rho} u^{(2)}(\tilde{x}, \tilde{z})=\int_{\mathbb{R}^{2}} \widehat{\bar{\rho} u^{(2)}} e^{i(\kappa \tilde{x}+\mu \tilde{z})} d \kappa d \mu .
$$

In particular, in fixed coordinates the vertical profile of the induced flow through the center of the translating wave packet is

$$
U(z, t)=u^{(2)}\left(x=c_{g_{x}} t, z\right),
$$

which is referred to as the "local wave-induced mean flow."
Figure 1a shows the wave-induced momentum given by (20). Qualitatively, this resembles the bow wake-like flow structure predicted by Bretherton (1969) and shown by van den Bremer and Sutherland (2014, their Fig. 2) for two-dimensional Boussinesq wave packets. Figure $1 \mathrm{~b}$ shows three vertical profiles of the waveinduced momentum through the horizontal center of the wave packet $\left(\tilde{x}=x-c_{g_{x}} t=0\right)$. The solid black curve is the profile determined by fast Fourier transforms applied to (20). The dashed red curve is computed from the integral given by (B4). That the curves overlap demonstrates the agreement among the results of the fast Fourier transform method described above and the residue theory-based method described in appendix $\mathrm{B}$. The dotted black curve is that computed for an effectively Boussinesq fluid, for which $H_{\rho}=1000 k^{-1}$. This profile is symmetric about $z=0$, with opposite sign above and below. We find that the wave-induced momentum of its anelastic counterpart is larger in magnitude on its trailing flank than its leading flank. It should be kept in mind, however, that the positive induced flow on its leading flank is larger than the magnitude of the negative flow on the trailing flank as a consequence of $\bar{\rho}$, given by (3), decreasing exponentially with height.

Although it is not explicit in (18), the induced flow $U(z, t)$ is $O\left(\alpha^{2} \varepsilon\right)$ as a consequence of the divergent flux induced flow forming a long wave whose energy is spread far from the wave packet itself (van den Bremer and Sutherland 2014, 2018). In contrast the flow $U_{1 \mathrm{D}}$ induced by horizontally periodic waves, given by (6), extends over the entire wave packet and is $O\left(\alpha^{2}\right)$. Also, consistent with the hydrostatic approximation for long waves, one finds $\|W\| \ll\|U\|$, in which $W$ is the vertical component of the induced flow (van den Bremer and Sutherland 2014). These properties will be exploited in the derivation of the nonlinear Schrödinger equation in the next section.

\section{b. Nonlinear Schrödinger equation}

Having established the expression for the waveinduced mean flow for horizontally and vertically localized anelastic internal gravity wave packets and its scaling in terms of $\alpha$ and $\varepsilon$, we can derive the nonlinear Schrödinger equation describing the moderately large amplitude evolution of such waves due to the induced flow which acts to Doppler-shift the waves. From the vorticity equation given by (8), we replace $\Psi$ with the 

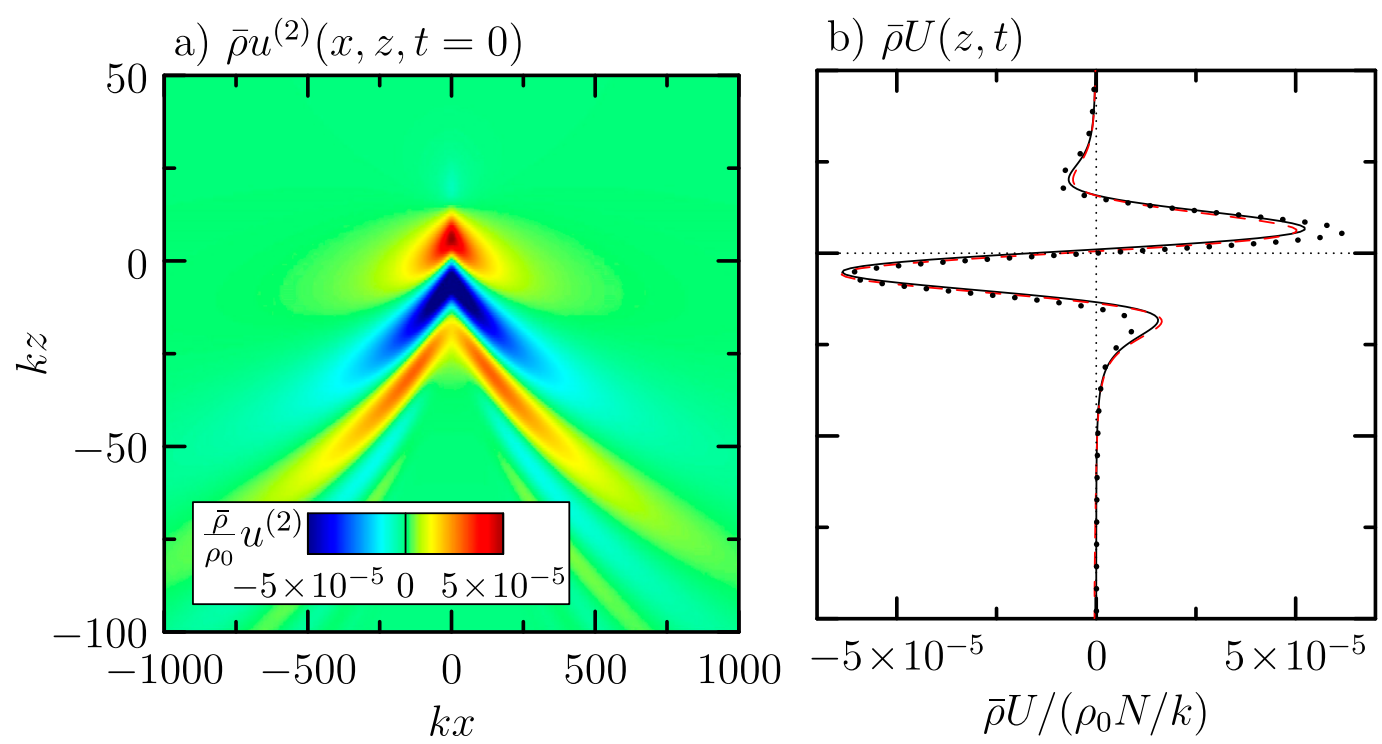

FIG. 1. (a) Initial density-scaled two-dimensional horizontal flow field, $\bar{\rho} u^{(2)}(x, z, t=0)$, induced by a Gaussian quasi-monochromatic wave packet via (20) and (b) its associated vertical profile of the wave-induced momentum, $\bar{\rho} U(z, t=0)$ through the horizontal center of the wave packet. The wave packet is initialized according to (30) with $m=-0.4 k, A_{0}=0.05 k^{-1}, \sigma_{x}=\sigma_{z}=10 k^{-1}$, and $H_{\rho}=10 k^{-1}$. In (b), the solid curve is found by taking a vertical slice through $x=0$ of $u^{(2)}$. The red dashed line is computed via the integral equation given by (B4). The black dotted curve is computed from (B4) in the near-Boussinesq case $H_{\rho}=1000 \mathrm{k}^{-1}$.

total field, that is, $\Psi_{T}=\bar{\Psi}(z, t)+\Psi(x, z, t)$, in which $\bar{\Psi}(z, t)$ is the $O\left(\alpha^{2} \varepsilon\right)$ induced mass streamfunction given by (17) evaluated at $\tilde{x}=0$ and $\Psi$ is the fluctuation mass streamfunction. We exploit here the fact that the waveinduced mean flow varies on a much wider scale than the packet itself, so that its value across the wave packet may be approximated by a constant corresponding to the value at the center of the wave packet $\tilde{x}=0$. In the resulting expressions, $z$ derivatives of $\bar{\Psi}$ are replaced by $-\bar{\rho} U$ according to (9a) and $x$ derivatives of $\bar{\Psi}$ are neglected under the approximation that $\|W\| \ll\|U\|$, as a consequence of working in two dimensions.

As with the derivation of the wave-induced mean flow, we assume the fluctuation mass streamfunction and vertical displacement fields have the form of quasimonochromatic wave packets, expressed as

$$
\begin{aligned}
\Psi & =\frac{1}{2} A_{\Psi}(x, z, t) e^{i \varphi-z / 2 H_{\rho}}+\text { c.c. } \\
\xi & =\frac{1}{2} A_{\xi}(x, z, t) e^{i \varphi+z / 2 H_{\rho}}+\text { c.c. },
\end{aligned}
$$

where $\varphi=k x+m z-\omega t$ is the phase, and c.c. denotes the complex conjugate so that $\Psi$ and $\xi$ are expressed explicitly as real fields. Substituting (22) and (23) into the equation for $\Psi$ and $\xi$, given by (11), extracting only those terms having the factor $e^{i \varphi}$ (i.e., those terms modeling the wavelike behavior we wish to capture), we are left with

$$
\begin{aligned}
& \partial_{t}\left(\nabla^{2}+\frac{1}{H_{\rho}} \partial_{z}\right) \Psi+U \partial_{x} \nabla^{2} \Psi+\frac{1}{H_{\rho}} U \partial_{x z} \Psi \\
& -\left(\partial_{x} \Psi\right)\left(\partial_{z z} U\right)-\frac{1}{H_{\rho}}\left(\partial_{x} \Psi\right)\left(\partial_{z} U\right)=-N^{2} \bar{\rho} \partial_{x} \xi
\end{aligned}
$$

in which $\partial_{x} \Psi=e^{i \varphi}\left(\partial_{x}+i k\right) A_{\Psi}, \quad \partial_{z} \Psi=e^{i \varphi}\left[\partial_{z}+i m-\right.$ $\left.1 /\left(2 H_{\rho}\right)\right] A_{\Psi}, \quad \partial_{t} \Psi=e^{i \varphi}\left(\partial_{t}-i \omega\right) A_{\Psi}, \quad$ and $\partial_{x} \xi=e^{i \varphi}\left(\partial_{x}+\right.$ ik) $A_{\xi}$.

Likewise, (7) is written in terms of $\bar{\Psi}, \Psi$, and $\xi$, and we extract terms from (7) containing the factor $e^{i \varphi}$ to give

$$
\left[\partial_{t}-i \omega+U\left(\partial_{x}+i k\right)\right] A_{\xi}=\frac{1}{\bar{\rho}}\left(\partial_{x}+i k\right) A_{\Psi} .
$$

Parameter $A_{\xi}$ may be straightforwardly eliminated from (24) and (25), although the resulting equation for $A_{\Psi}$ alone is algebraically cumbersome. Finally, this expression is recast explicitly in terms of the slow variables $(x, z, t) \rightarrow\left[X=\varepsilon\left(x-c_{g_{z}} t\right), Z=\varepsilon\left(z-c_{g_{z}} t\right), T=\varepsilon^{2} t\right]$. In this translating frame the time evolution appears at $O\left(\varepsilon^{2}\right)$, describing the dispersion of the wave packet.

Assuming $A_{\Psi}$ can be written as a perturbation expansion of the form $A_{\Psi}=\alpha\left(B_{0}+\varepsilon B_{1}+\varepsilon^{2} B_{2}+\cdots\right)$ and likewise $U=\alpha^{2} \varepsilon\left(V_{0}+\varepsilon V_{1}+\cdots\right)$, we extract the $O\left(\alpha^{r} \varepsilon^{s}\right)$ parts of the nonlinear equation for $A_{\Psi}$ up to and including the combined order $r+s=4$. We assume $\alpha \sim \varepsilon$. The $O\left(\alpha \varepsilon^{0}\right)$ equation recovers the linear dispersion relation for anelastic internal gravity waves. The $O(\alpha \varepsilon)$ equation 
yields the trivial result $0=0$ as a consequence of working in a frame of reference translating at the group velocity. The $O\left(\alpha \varepsilon^{2}\right)$ equation yields the linear Schrödinger equation describing leading-order dispersion of wave packets in two spatial dimensions in a frame of reference translating at the group velocity,

$$
\partial_{T} B_{0}=i\left(\frac{1}{2} \omega_{k k} \partial_{X X}+\omega_{k m} \partial_{X Z}+\frac{1}{2} \omega_{m m} \partial_{Z Z}\right) B_{0} .
$$

Here the subscripts on $\omega$ denote partial derivatives with respect to wavenumber components. The $O\left(\alpha^{3} \varepsilon+\alpha \varepsilon^{3}\right)$ equation contains both leading- and next-order linear dispersion terms as well as the nonlinear term representing Doppler shifting of the waves by their induced flow at the center of the wave packet where $X=\tilde{x}=0$,

$$
\begin{aligned}
\partial_{T} B_{1}= & i\left(\frac{1}{2} \omega_{k k} \partial_{X X}+\omega_{k m} \partial_{X Z}+\frac{1}{2} \omega_{m m} \partial_{Z Z}\right) B_{1} \\
& +\left(\frac{1}{6} \omega_{k k k} \partial_{X X X}+\frac{1}{2} \omega_{k k m} \partial_{X X Z}+\frac{1}{2} \omega_{k m m} \partial_{X Z Z}\right. \\
& \left.+\frac{1}{6} \omega_{m m m} \partial_{Z Z Z}\right) B_{0}-i k V_{0} B_{0}
\end{aligned}
$$

Recombining all orders and returning to a fixed frame of reference yields the nonlinear Schrödinger equation in its final form,

$$
\begin{aligned}
\partial_{t} A_{\Psi}= & -\left(\omega_{k} \partial_{x}+\omega_{m} \partial_{z}\right) A_{\Psi}+i\left(\frac{1}{2} \omega_{k k} \partial_{x x}+\omega_{k m} \partial_{x z}\right. \\
& \left.+\frac{1}{2} \omega_{m m} \partial_{z z}\right) A_{\Psi}+\left(\frac{1}{6} \omega_{k k k} \partial_{x x x}+\frac{1}{2} \omega_{k k m} \partial_{x x z}\right. \\
& \left.+\frac{1}{2} \omega_{k m m} \partial_{x z z}+\frac{1}{6} \omega_{m m m} \partial_{z z z}\right) A_{\Psi}-i k U A_{\Psi} .
\end{aligned}
$$

Explicit expressions for the linear dispersion relation $\omega$ and its derivatives are summarized in Table 2.

The grouped terms on the right-hand side of (28) respectively represent linear advection at the group velocity, leading-order linear dispersion, and next-order linear dispersion. The mixed derivative terms in these groups represent what we refer to as "oblique" dispersion. The last (nonlinear) term on the right-hand side of (28) represents the Doppler shifting of the waves by the induced flow $U=u^{(2)}\left(x=c_{g_{x}} t, z, t\right)$, given at $O\left(\alpha^{2} \varepsilon\right)$ by (18). Here $u^{(2)}$ is explicitly a function of time because $A_{\Psi}$ evolves in time according to (28).

The linear part of (28) is identical to that of the twodimensional nonlinear Schrödinger equation derived by Shrira [1981, their Eq. (20)], for Boussinesq internal gravity wave packets in three spatial dimensions in which dependence on the spanwise coordinate is significantly weaker than on $x$ and $z$. The key difference between
(28) herein and (20) of Shrira (1981) is the form of the nonlinear part of the respective equations. Shrira's nonlinear terms are proportional to $A\left(\mathbf{c}_{g} \cdot \nabla A^{\star}\right)-|A|^{2} \mathbf{c}_{g} \cdot \nabla A$, where the star denotes the complex conjugate of $A$. Tabaei and Akylas (2007) derived a wave packet evolution equation that reduces to a nonlinear Schrödinger equation in the absence of modulations in the $x$ direction [cf. (66) and (72) in that work]. Like (28), the nonlinearity therein arises from interactions with the waves and their induced mean flow. However, unlike the equations of Tabaei and Akylas, our nonlinear Schrödinger equation retains sensitivity to horizontal modulations, although its effect is significantly weaker than the effect of vertical modulations.

The anelastic nonlinear Schrödinger equation for twodimensional internal gravity wave packets given by (28) differs from its one-dimensional (horizontally periodic) counterpart [cf. (26) in DS11] in three key ways. Being horizontally localized means the wave packet can disperse horizontally as well as vertically, as expressed by the $x$-derivative terms. The wave-induced mean flow $U$ is qualitatively different from its horizontally periodic counterpart: rather than being unidirectional with peak value where the amplitude envelope peaks, $U$ is positive on the leading flank and negative on the trailing flank. Finally, because $U$ for a two-dimensional wave packet is $O\left(\alpha^{2} \varepsilon\right)$, not $O\left(\alpha^{2}\right)$ as is the case for one-dimensional wave packets, the nonlinear term in (28) balances the dispersion terms with three spatial derivatives (for $\varepsilon \sim \alpha$ ). In DS11, those terms were necessarily balanced by the vertical shear of the wave-induced mean flow, whereas the Doppler-shift term balanced leading-order dispersion.

Taking the horizontal extent of the wave packet $\sigma_{x}$ to be infinitely large, the wave packet amplitude envelope becomes horizontally uniform and so all terms containing at least one $x$ derivative in (28) vanish. Also taking the Boussinesq limit $H_{\rho} \rightarrow \infty$, the linear part of the resulting equation recovers the linear part of that derived by Sutherland [2006b , their (2.10)]. Despite the anticipated reduction of the two-dimensional nonlinear Schrödinger equation, (28), to the one-dimensional nonlinear Schrödinger equation in the $\sigma_{x} \rightarrow \infty$ limit, it is important to note that the induced flow $U$ is different in these two formulas, given by (6) in the one-dimensional case and by (18)-(21) in the two-dimensional case. The latter formulas do not reduce to (6) in the limit of horizontally periodic waves because the divergent flux induced flow is itself divergent for horizontally localized waves and, consequently, the induced flow responds to excite long waves whose flow changes sign from the leading to trailing flank of the wave packet (Tabaei and Akylas 2007; van den Bremer and Sutherland 2014, 2018). 
TABLE 2. Expressions for the linear dispersion relation $\omega$ and its derivatives up to third order. In each expression, subscripts on $\omega$ denote partial derivatives and $K^{2}=k^{2}+m^{2}+1 / 4 H_{\rho}^{2}$. The frequency and horizontal wavenumber $k$ are taken to be positive, and the vertical wavenumber $m$ is taken to be negative, corresponding to waves that propagate upward and to the right.

\begin{tabular}{rlrl}
\hline \hline $\begin{array}{c}\text { Dispersion relation and } \\
\text { its derivatives }\end{array}$ & \\
\hline$\omega=\frac{N}{K} k$ & $\omega_{k k k}=-3 \frac{N}{K^{7}}\left(K^{2}-k^{2}\right)\left(K^{2}-5 k^{2}\right)$ \\
$c_{g_{x}}=\omega_{k}=\frac{N}{K^{3}}\left(K^{2}-k^{2}\right)$ & $\omega_{k k m}=3 \frac{N}{K^{7}} k m\left(3 K^{2}-5 k^{2}\right)$ \\
$c_{g_{z}}=\omega_{m}=-\frac{N}{K^{3}} k m$ & $\omega_{k m m}=-\frac{N}{K^{7}}\left[K^{2}\left(3 K^{2}-5 k^{2}\right)\right.$ \\
$\omega_{k k}=-3 \frac{N}{K^{5}} k\left(K^{2}-k^{2}\right)$ & $\omega_{m m m}=3 \frac{N}{K^{7}} k m\left(3 K^{2}-5 m^{2}\right)$ \\
$\omega_{k m}=-\frac{N}{K^{5}} m\left(K^{2}-3 k^{2}\right)$ & \\
$\omega_{m m}=-\frac{N}{K^{5}} k\left(K^{2}-3 m^{2}\right)$ & &
\end{tabular}

Modulational stability or instability refers to whether weakly nonlinear effects cause a wave packet to broaden and decay in amplitude (stability), or to narrow and grow in amplitude (instability). In the context of internal waves, the key to modulational stability or instability is the effect of Doppler shifting on the vertical group velocity by the nonlinear term in the Schrödinger equation (28). In particular, for the upward- and rightwardpropagating waves under consideration here, the wave-induced mean flow $U$ Doppler shifts the frequency $\omega$ toward larger values if $U>0$, and toward smaller values if $U<0$. In turn, Doppler shifting causes a change in the vertical group speed: locally increasing this speed causes wave spreading (modulational stability), and hence relative amplitude decay, whereas locally decreasing this speed causes wave accumulation (modulational instability), hence relative amplitude growth (see also Sutherland 2010, their section 4.2.4; Whitham 1974). Mathematically, wave packets are modulationally unstable if the product of the coefficients of the nonlinear term (the wave-induced mean flow) and the dispersive term $\omega_{m m}$ is negative. For one-dimensional wave packets, this occurs for waves with frequency greater than that for waves with the fastest vertical group velocity (for which $\omega_{m m}=0$ ). For Boussinesq wave packets this transition occurs at the critical frequency $\omega_{*}=(2 / 3)^{1 / 2} N$ with corresponding critical wavenumber $m_{*}=2^{-1 / 2} k$. For two-dimensional wave packets, because $k U$ in the last term of (28) changes sign from the leading flank to the trailing flank, twodimensional wave packets are always modulationally unstable, consistent with the findings of Tabaei and Akylas (2007), who performed a linear stability analysis to demonstrate this. Specifically, where $k U \omega_{m m}<0$ relative amplitude growth occurs due to wave accumulation and where $k U \omega_{m m}>0$ relative amplitude decay occurs due to wave spreading. Whether the leading or trailing flank exhibits growth is determined by the magnitude of the critical relative vertical wavenumber $\tilde{m}_{*}$ set by the condition $\omega_{m m}=0$. Explicitly,

$$
\tilde{m}_{*}=\frac{m_{*}}{k} \equiv \frac{1}{\sqrt{2}}\left(1+\frac{1}{4 k^{2} H_{\rho}^{2}}\right)^{1 / 2} .
$$

If $|m / k|<\tilde{m}_{*}$, wave packets accumulate on the leading flank [where $U(z, t)>0$ ], while the trailing flank, which tends to spread vertically, inevitably runs into the leading flank. If $|m / k|>\tilde{m}_{*}$, the leading flank spreads out while the trailing flank accumulates. In both cases, the wave packet is unstable.

\section{Numerical simulation and analysis methods}

Here we discuss the numerical methods for the solution of the weakly and fully nonlinear equations of motion and we present the methods used to analyze the results of the simulations.

\section{a. Weakly nonlinear simulations}

The weakly nonlinear code solved (28) on a finitedifference grid. Associated with this domain was an equivalent doubly periodic Fourier space domain in which the wave-induced mean flow was computed, using the method described in section $2 \mathrm{a}$, with only the value $U(z, t)=u^{(2)}\left(x=c_{g_{x}} t, z, t\right)$ used in (28).

The scales for time and the domain size were set respectively by fixing $N=1$ and $k=1$. All simulations were run on a domain of size $[-1608.6 \leq k x \leq 1608.6] \times$ [ $-250 \leq k z \leq 150]$ with 2049 grid points in the horizontal and 513 grid points in the vertical. The horizontal domain was chosen to be large enough for the long waves induced by the wave packet to be of negligible amplitude at the left and right boundaries, hence minimizing boundary effects due to the doubly periodic Fourier space domain in which the induced flow field was computed. The vertical domain was similarly chosen to be deep enough that the induced long wave was of negligible amplitude at the bottom boundary, and tall enough to allow the wave packet to propagate well above its initial vertical position (always set to $z_{0}=0$ ) without interacting with the top boundary. Figure 1a illustrates such a wide and deep domain.

The simulations were initialized with a bivariate Gaussian wave packet whose initial vertical displacement amplitude envelope was prescribed to be 
TABLE 3. The frequency $\omega$; horizontal and vertical group velocities $c_{g_{x}}=\omega_{k}$ and $c_{g_{z}}=\omega_{m}$, respectively; and higher-order $k$ and $m$ derivatives of $\omega$ computed using $H_{\rho}=10 \mathrm{k}^{-1}$ for the range of values of $\mathrm{m} / \mathrm{k}$ considered in the weakly nonlinear simulations.

\begin{tabular}{rrrrrrrrrrr}
\hline \hline$m / k$ & $\omega / N$ & $c_{g_{x}} \frac{k}{N}$ & $c_{g_{z}} \frac{k}{N}$ & $\omega_{k k} \frac{k^{2}}{N}$ & $\omega_{k m} \frac{k^{2}}{N}$ & $\omega_{m m} \frac{k^{2}}{N}$ & $\omega_{k k k} \frac{k^{3}}{N}$ & $\omega_{k k m} \frac{k^{3}}{N}$ & $\omega_{k m m} \frac{k^{3}}{N}$ & $\omega_{m m m} \frac{k^{3}}{N}$ \\
\hline-0.4 & 0.928 & 0.130 & 0.319 & -0.335 & -0.504 & -0.468 & 1.105 & 1.072 & 0.170 \\
-0.7 & 0.819 & 0.270 & 0.384 & -0.543 & -0.388 & -0.008 & 1.126 & 0.270 & -0.717 \\
-1.4 & 0.581 & 0.385 & 0.275 & -0.390 & -0.004 & 0.193 & 0.268 & -0.365 & -0.266 & -1.048 \\
\hline
\end{tabular}

$$
A(x, z, 0)=A_{0} \exp \left[-\frac{1}{2}\left(\frac{x^{2}}{\sigma_{x}^{2}}+\frac{z^{2}}{\sigma_{z}^{2}}\right)\right]
$$

where $\sigma_{x}$ and $\sigma_{z}$, respectively, were the horizontal and vertical extent of the wave packet. For all simulations, the initial vertical displacement amplitude $A_{0}$ was set to be $0.05 k^{-1}$, so that our results could be compared with those of DS11. Although the nonlinear Schrödinger equation describes the mass-streamfunction envelope, we initially prescribed the vertical displacement as a more physically intuitive quantity. The leading-order polarization relations, provided in the center column of Table 1, were then used to initialize the massstreamfunction amplitude envelope,

$$
A_{\Psi}(x, z, 0)=-\frac{\omega}{k} A(x, z, 0) .
$$

For any time step, centered second-order finite differences were used to compute all spatial derivatives on the right-hand side of (28). The coefficients of the (linear) advection and dispersion terms are given nondimensionally for a range of vertical wavenumbers in Table 3 . The contribution from the wave-induced mean flow was then multiplied by $-i k A_{\Psi}$ and added to the linear part. Time stepping was performed using a leapfrog scheme, with an Euler backstep taken every 20 steps for numerical stability. For all simulations, time was advanced by a small increment of $\Delta t=0.005 N^{-1}$.

The code used the leading-order polarization relations to output the vertical displacement field, the modulus of the vertical displacement amplitude $\left|A_{\xi}(x, z)\right|$, the waveinduced momentum $\bar{\rho} u^{(2)}$, and its corresponding vertical profile at the horizontal center of the wave packet $\bar{\rho} U(z, t)=\bar{\rho} u^{(2)}(\tilde{x}=0, z, t)$.

\section{b. Fully nonlinear simulations}

The 2D fully nonlinear code solves an adaptation of (1) recast in terms of the spanwise vorticity $\zeta$ and vertical displacement $\xi$. For numerical stability the actual vorticity, vertical displacement, and velocity fields are scaled to remove the effects of linear anelastic growth according to

$$
\left(\zeta_{s}, \xi_{s}, u_{s}, w_{s}\right)=(\zeta, \xi, u, w) e^{-z / 2 H_{\rho}} .
$$

Here, the subscript $s$ denotes the scaled fields. Thus, the equations for the scaled vorticity and vertical displacement are

$$
\begin{aligned}
& \frac{\partial \zeta_{s}}{\partial t}=-e^{z / 2 H_{\rho}}\left(\mathbf{u}_{s} \cdot \nabla \zeta_{s}+\frac{3}{2 H_{\rho}} w_{s} \zeta_{s}\right)+N^{2} \frac{\partial \xi_{s}}{\partial x}+C_{\zeta} \mathscr{D} \zeta_{s} \\
& \frac{\partial \xi_{s}}{\partial t}=-e^{z / 2 H_{\rho}}\left(\mathbf{u}_{s} \cdot \nabla \xi_{s}+\frac{1}{H^{*}} w_{s} \xi_{s}\right)+w_{s}+C_{\xi} \mathscr{D} \xi_{s}
\end{aligned}
$$

where we have set $1 / H^{*}=1 /\left(2 H_{\rho}\right)+1 / H_{\theta}$ for notational convenience. The last terms in (33) and (34) include the effects of viscosity and diffusion through a horizontally spectral, vertically real Laplacian-like operator $\mathscr{D}$ acting at all vertical levels and only on horizontal wavenumbers greater than a specified wavenumber $k_{d}$. This damps numerical noise while not acting to attenuate the waves. For all simulations, we set $k_{d}=3 k_{n} / 2$, where $k_{n}=256$ is the number of waves in the domain. The values of the coefficients $C_{\zeta}=C_{\xi}=10^{-3} \mathrm{Nk}^{-2}$ were chosen such that the wave dynamics were negligibly affected.

The time and domain size were set by fixing $k=1$ and $N=1$. The domain is real in the vertical and spectral in the horizontal with periodic boundary conditions in the horizontal and free-slip upper and lower boundary conditions. The vertical domain was set so that $-30 \leq k z \leq 150$ with 1024 grid points. The extent of the horizontal domain $L_{x}$ was set by specifying the number of horizontal wavelengths, $k_{n} \equiv L_{x} /(2 \pi / k)$, that can exist in the domain. Hence, $|k x| \leq \pi k_{n}$. In all simulations $k_{n}=256$ and so $|k x| \leq 804.3$. This domain was discretized by 8192 wavenumbers in Fourier space.

The simulations were initialized with the scaled mass streamfunction, whose amplitude envelope was given by (30). The amplitude $A_{\Psi_{0}}=\left|-(\omega / k) A_{0}\right|$ was computed using the prescribed value of $A_{0}=0.05 \mathrm{k}^{-1}$. The initial spanwise vorticity $\zeta_{s}$ was defined by numerically differentiating $\Psi_{s}(x, z, 0)$ according to $(10)$, and the initial vertical displacement $\xi_{s}$ was defined from the polarization relations, as in the second column of Table 1.

It is physically realistic to include the induced flow when initializing the fully nonlinear simulations. However, very large domains are necessary to compute the induced flow accurately. Because only the flow over the 
extent of the wave packet is important to govern the wave packet evolution, and because this flow begins to develop outward and downward from the center of the translating wave packet shortly after initialization, the fully nonlinear simulations were initialized without an induced flow. In particular, the time scale $t_{\sigma}$ on which the induced flow spreads over the horizontal extent of the wave packet is predicted by the ratio of the horizontal extent $\sigma_{x}$ to the horizontal group speed of the induced long wave $\bar{c}_{g_{x}}$. Building on the argument of van den Bremer and Sutherland (2014, their section III C), this is determined from the relations

$$
\begin{aligned}
\bar{\omega} & =\frac{N \bar{k}}{|\bar{m}|}, \\
|\bar{m}| & =\frac{2 \pi}{\sigma_{z}}, \\
\bar{c}_{p_{x}} \approx \bar{c}_{g_{x}} & =\frac{\bar{\omega}}{\bar{k}},
\end{aligned}
$$

where the bars denote that these apply to the horizontally long induced internal waves. Respectively, these identities are the dispersion relation for long waves, the magnitude of the vertical wavenumber, assumed to scale with the vertical extent $\sigma_{z}$ of the wave packet, and the horizontal phase velocity, which approximately equals the horizontal group velocity for long waves. It follows that

$$
t_{\sigma}=\frac{\sigma_{x}}{\bar{c}_{g_{x}}}=\frac{2 \pi \sigma_{x}}{N \sigma_{z}} .
$$

This shows that the induced flow became well developed over a wave packet width $\sigma_{x} \sim \sigma_{z}$ after one buoyancy period. As such, neglecting to initialize the induced flow had negligible influence on the long-time behavior of the wave packet. Furthermore, its neglect meant that the lower vertical domain limit $\left|z_{\min }\right|$ could be set to be much smaller than for the weakly nonlinear simulations that were initialized with the predicted induced flow superimposed on the wave packet.

At a given time step, $\zeta_{s}$ was used to compute the mass streamfunction via the relation (10). This involved matrix inversion in which $\zeta_{s}$ and the differential operators in (10) were Fourier decomposed in the horizontal. In turn, the scaled mass streamfunction was used to compute the scaled horizontal and vertical velocities via the relations (9a) and (9b), using fast Fourier transforms in the horizontal and centered, second-order finite differences in the vertical. The advection terms were evaluated by horizontally inverse fast Fourier transforming these and the $\zeta_{s}$ and $\xi_{s}$ fields, multiplying in real space, and horizontally fast Fourier transforming the product.
Using the leapfrog scheme, the scaled vertical displacement and vorticity fields were advanced in time by a small increment, taken to be $\Delta t=0.0125 \mathrm{~N}^{-1}$ for all simulations. An Euler backstep was taken every 20 time steps for numerical stability.

\section{c. Analysis methods}

From the fully nonlinear code, the actual and scaled vertical displacement fields were output after horizontally inverse fast Fourier transforming the computed $\xi_{s}$ field. The actual and scaled horizontal velocity fields were computed from the relation (9a), the result of which was horizontally inverse fast Fourier transformed. The wave packet was filtered to reveal only the induced flow. This was done through a low-pass filter by retaining horizontal wavenumbers smaller than $k_{n} / 2$ and inverse transforming the result. In particular, vertical profiles of the wave-induced mean flow through the center of the translating wave packet were determined by low-pass Fourier filtering the horizontal velocity field, and extracting the profile where $\tilde{x}=c_{g_{x}} t$.

We gain insight about the time scales on which weakly and fully nonlinear effects develop by considering time series of the $L^{2}$ norms of the wave-induced momentum at the horizontal centerline of the translating wave packet $\tilde{x}=0$. This is defined nondimensionally as

$$
\|\widetilde{M}(t)\| \equiv \frac{\|f(\tilde{z}, t)\|}{\left\|f\left(\tilde{z}, t_{*}\right)\right\|}=\frac{\left[\int_{\tilde{z}_{\min }}^{\tilde{z}_{\max }} f^{2}(\tilde{z}, t) d z\right]^{1 / 2}}{\left[\int_{\tilde{z}_{\min }}^{\tilde{z}_{\max }} f^{2}\left(\tilde{z}, t_{*}\right) d z\right]^{1 / 2}} .
$$

Here $f(\tilde{z}, t)=\bar{\rho} U(\tilde{z}, t)$ is the wave-induced momentum in a frame of reference translating with the wave packet. For the weakly nonlinear simulations, $\|f(\tilde{z}, t)\|$ is normalized by the $L^{2}$ norm of the wave-induced momentum at $t_{*}=0$, predicted by setting $\tilde{x}=0$ in (B4). For the fully nonlinear simulations, $\|f(\tilde{z}, t)\|$ is normalized by the $L^{2}$ norm of the wave-induced momentum at $t_{*}=t_{\sigma}$, with $t_{\sigma}$ given by (36). This is the time at which the induced long wave has developed across the horizontal extent of the wave packet.

For comparison of our results with those of DS11, it is useful to estimate the height at which the interaction of the waves with their induced mean flow becomes significant. Following the approach of DS11, we predict the waveinduced mean flow magnitude above which weakly nonlinear effects become nonnegligible by equating the magnitudes of the nonlinear term and the smallest of the linear advection terms in the nonlinear Schrödinger equation, (28). Explicitly, these are comparable when the magnitude of the wave-induced mean flow is $|U| \sim \varepsilon \min \left\{\left|c_{g_{x}}\right|,\left|c_{g_{z}}\right|\right\}$. For Boussinesq wave packets, van den Bremer and Sutherland (2018) derived an asymptotic approximation 
for the maximum horizontal velocity of the induced long wave [cf. (3.27) in that work]. For spanwise-uniform wave packets, we somewhat heuristically incorporate linear anelastic effects by dividing their result by $\bar{\rho} / \rho_{0}=e^{-z / H_{\rho}}$, and replacing instances of $|\mathbf{k}|$ with $K$ to predict

$$
\left.\max _{\tilde{z}} U\right|_{\tilde{x}=y=0}=\frac{1}{2} N K A_{0}^{2}\left(1.45 \frac{k^{2}|m|}{K^{3}} \varepsilon \frac{\sigma_{x}^{2}}{\sigma_{z}^{2}} e^{z / H_{\rho}}\right) .
$$

Equating the right-hand side of (38) with $\varepsilon \min \left\{\left|c_{g_{x}}\right|,\left|c_{g_{z}}\right|\right\}=$ $\left(N / K^{3}\right) \min \left\{K^{2}-k^{2},|k m|\right\}$, the height $z_{\Delta}$ at which we expect weakly nonlinear effects to become significant is given nondimensionally by

$$
\begin{aligned}
\frac{z_{\Delta}}{H_{\rho}}= & \ln \left(\frac{1.38 \sigma_{z}^{2}}{A_{0}^{2} k^{2}|m| K \sigma_{x}^{2}}\right) \\
& +\left\{\begin{array}{cc}
\ln \left(K^{2}-k^{2}\right), & |k|>|m|+1 /\left(4|m| H_{\rho}^{2}\right), \\
\ln (|k m|), & |k|<|m|+1 /\left(4|m| H_{\rho}^{2}\right) .
\end{array}\right.
\end{aligned}
$$

Assuming the vertical phase speed of the induced long wave is equal to the vertical group speed of the wave packet, the predicted time at which weakly nonlinear effects become significant is given by

$$
t_{\Delta}=\frac{z_{\Delta}}{c_{g_{z}}} .
$$

Generally in simulations, wave overturning was diagnosed by computing the minimum value of the total squared buoyancy frequency, $N_{T}^{2}=N^{2}+\Delta N^{2}$, where

$$
\Delta N^{2} \equiv \frac{g}{\bar{\theta}} \frac{\partial \theta}{\partial z} \approx-N^{2} \frac{\partial \xi}{\partial z} .
$$

Overturning occurs where $N_{T}^{2}<0$. At each time step, the fully nonlinear code found the minimum value of $N_{T}^{2}$ in the domain. In practice, the first time $t_{b}$ for which this quantity was negative was taken to be the time of wave overturning. The corresponding vertical location is $z_{b}$, where $N_{T}^{2}<0$ was taken to be the overturning location.

Linear anelastic theory predicts the overturning height to occur where $\left|\partial_{z} \xi\right|=1$. Explicitly,

$$
z_{b, L}=2 H_{\rho} \ln \left[\frac{1}{A_{0}|m|}\left(1+\frac{1}{4 H_{\rho}^{2} m^{2}}\right)^{-1 / 2}\right] .
$$

\section{Weakly and fully nonlinear simulation results and their comparison}

In each simulation discussed below, the values of horizontal wavenumber $k$ and buoyancy frequency $N$ were fixed, and we took the density scale height of the model atmosphere to be $H_{\rho}=10 k^{-1}$. Our primary focus is on Gaussian wave packets with initial horizontal extent $\sigma_{x}=10 k^{-1}$. However, simulations were also conducted by setting $\sigma_{x}=40 k^{-1}$. The choice of vertical wavenumbers, from $m=-0.4 k,-0.7 k$, and $-1.4 k$, spans the range studied by DS11 from modulationally unstable, marginally stable, and modulationally stable horizontally periodic wave packets. However, here the qualitatively different structure of the two-dimensional wave-induced mean flow renders the wave packets modulationally unstable in all cases, as shown and discussed below (and by Tabaei and Akylas 2007).

\section{a. Weakly nonlinear simulations}

A plot showing the weakly nonlinear evolution of a wave packet with $m=-0.4 k, A_{0}=0.05 k^{-1}, \sigma_{x}=10 k^{-1}$, and $H_{\rho}=10 k^{-1}$ is shown at four different times in Fig. 2 .

At $t=50 \mathrm{~N}^{-1}$ the vertical displacement amplitude envelope, shown in the center-left panel of Fig. 2a, has changed little from its initial Gaussian structure. The wave packet has translated up and rightward according to linear theory, evident in part by the small relative discrepancy between the locations of the observed and predicted peak envelope amplitudes, defined by

$$
\delta_{c} \equiv\left(\frac{x_{\text {peak }}-c_{g_{x}} t}{\sigma_{x}}, \frac{z_{\text {peak }}-c_{g_{z}} t}{\sigma_{z}}\right),
$$

in which $\left(x_{\text {peak }}, z_{\text {peak }}\right)$ is the location of the observed peak envelope amplitude. In Fig. $2 \mathrm{a},\left.\delta_{c}\right|_{t=50 N^{-1}} \approx-(0.02,0.03)$. The rightmost panel of Fig. 2a shows the vertical profile of the wave-induced momentum through the center of the translating wave packet. The induced flow, scaled by $\bar{\rho} / \rho_{0}=e^{-z / H_{\rho}}$, is nearly symmetric about the horizontal center of the wave packet with the magnitude of the negative flow, located on the trailing half of the wave packet, being slightly larger than that of the positive flow, located on the leading half.

At $t=100 N^{-1}$ (Fig. 2b) linear dispersion has caused the wave packet amplitude envelope to widen and stretch slightly in the horizontal and vertical directions, respectively. Oblique dispersion [involving dispersion terms in (28) with both $k$ and $m$ derivatives of $\omega$ ] has caused the wave packet to "tilt" clockwise. [No tilt of the amplitude envelope was observed in weakly nonlinear simulations (not shown) solved without the oblique dispersion terms]. As a consequence of this dispersion, the maximum value of the vertical displacement amplitude is somewhat smaller than that predicted by linear theory for periodic anelastic waves.

The results at this time indicate that weakly nonlinear effects have not yet begun significantly to modify the 

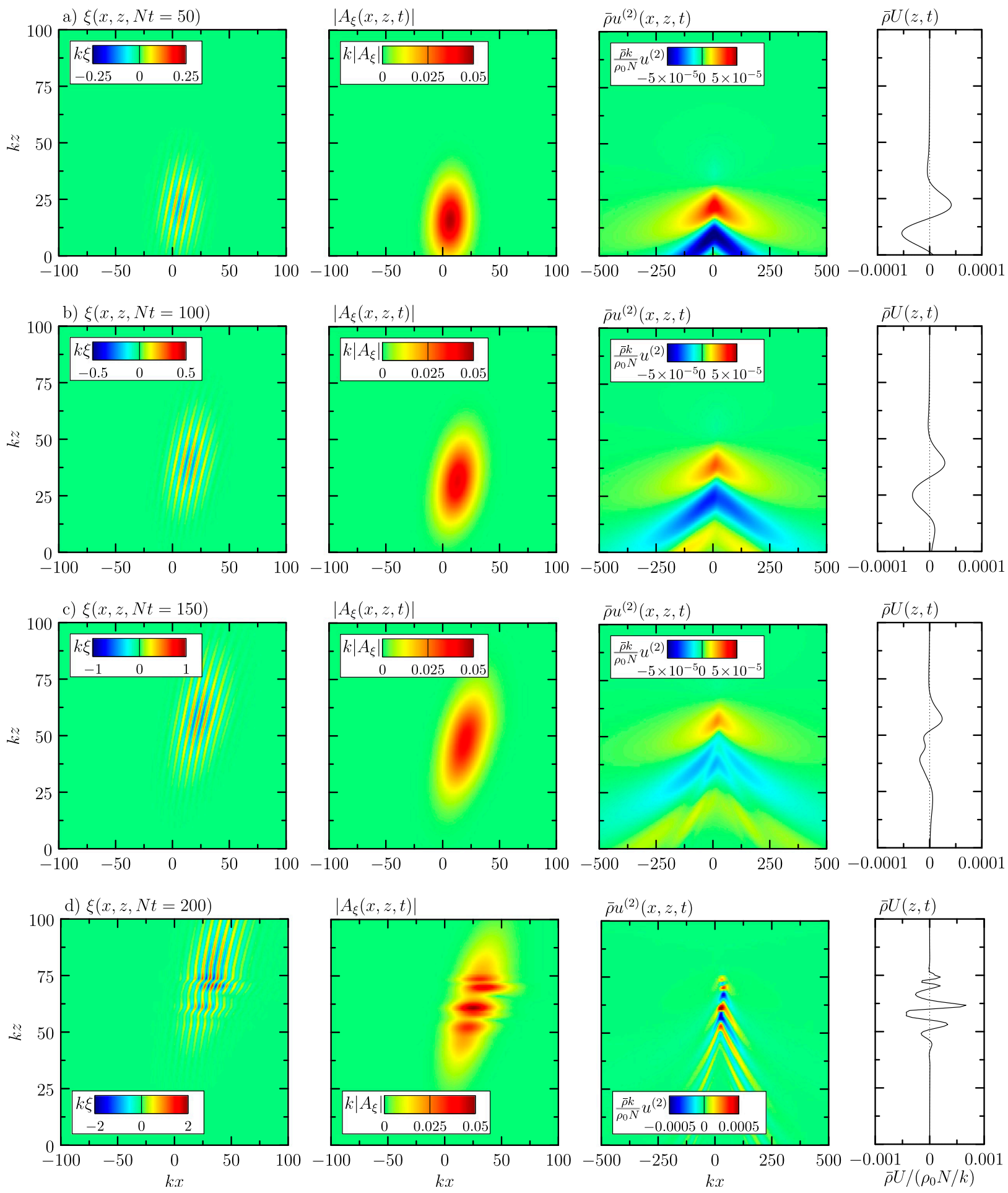

FIG. 2. Evolution of a weakly nonlinear internal gravity wave packet with $m=-0.4 k$ at times $t=$ (a) $50 N^{-1}$, (b) $100 N^{-1}$, (c) $150 N^{-1}$, and (d) $200 N^{-1}$. The wave packet is initialized according to (30) with $A_{0}=0.05 k^{-1}$ and $\sigma_{x}=\sigma_{z}=10 k^{-1}$ and propagates through an atmosphere with density scale height $H_{\rho}=10 \mathrm{k}^{-1}$. Each row shows (left) the vertical displacement, (center left) the modulus of the vertical displacement amplitude, (center right) the density-scaled induced horizontal flow field, and (right) a vertical profile of the wave-induced mean flow through the horizontal center of the translating wave packet. 
wave packet evolution, in contrast to the analogous simulation for horizontally periodic waves by DS11 (cf. Fig. $1 \mathrm{~b}$ in that work). This is due primarily to the scaling of the wave-induced mean flow for two-dimensional wave packets. Being of $O\left(\alpha^{2} \varepsilon\right)$, compared to $O\left(\alpha^{2}\right)$ for one-dimensional wave packets, the onset of weakly nonlinear effects requires the waves to grow to relatively larger amplitude and so takes more time for the waves to propagate vertically to correspondingly greater altitudes.

At $t=150 N^{-1}$ (Fig. 2c), oblique dispersion has continued to cause the wave packet to tilt clockwise. As a result, the wave-induced mean flow exhibits a slight horizontal asymmetry, with magnitudes in the region $\tilde{x}<0$ somewhat larger than those in the region $\tilde{x}>0$. (No such asymmetry was observed at this time in the corresponding simulation solved without the oblique dispersion terms). Also seen in the wave-induced mean flow is a narrowing of the bow wake-like structure below the center of the wave packet.

At $t=200 N^{-1}$ (Fig. 2d), weakly nonlinear effects have modulated the wave packet to such an extent that it might no longer justifiably be considered quasi monochromatic. Phase lines in the vertical displacement are tilted more strongly toward the vertical, with small areas near the center of the wave packet apparently tilting opposite their initial orientation. The discrepancy between the observed and predicted locations of the amplitude envelope peak is $\delta_{c} \approx-(0.08,0.29)$. The vertical translation of the wave packet has slowed due to Doppler shifting of the leading edge of the wave packet by the wave-induced mean flow. Finally, the waveinduced mean flow has degenerated into a series of positively and negatively flowing jets whose maximum velocities have increased by an order of magnitude from earlier times (Figs. 2a-c). Diagnostics presented later show that the wave packet is close to overturning at this time.

The relative wave-induced momentum $\widetilde{M}$, given by (37), for this simulation is shown as the solid curve in Fig. 3a. Because of dispersion, until $t \approx 170 N^{-1}$ the wave-induced momentum steadily decreases. Afterward, the momentum magnitude rapidly increases, indicating that weakly nonlinear effects have become significant. The predicted time at which this is expected to occur is indicated by the vertical line located at $t_{\Delta}=167 N^{-1}$, given by (40).

As another representation of the wave packet evolution, Fig. 4a shows the time series of the wave-induced momentum at the horizontal center of the wave packet $\left(\tilde{x}=x-c_{g_{x}} t=0\right)$ in the simulation with $m=-0.4 k$. The flow is plotted in a frame of reference translating at the vertical group velocity $\tilde{z}=z-c_{g_{z}} t$ and further normalized by the initial maximum value of the waveinduced momentum $\rho_{0} U_{0}=\max _{z}\{\bar{\rho} U(z, t=0)\}$.

Wave packet spreading due to dispersion is visible between approximately $t=100 N^{-1}$ and $t=150 N^{-1}$, shortly after which nonlinear effects become apparent and cause the wave-induced momentum at $\tilde{x}=0$ to degenerate into a series of positively and negatively flowing jets (see also Fig. 3a). In contrast to horizontally periodic waves (DS11), the jets advance in time toward both the leading and trailing edges of the wave packet, as opposed to the trailing edge alone. The observed weakly nonlinear evolution is due to Doppler shifting that causes the wave packet to narrow and peak on its leading flank where $U>0$ and $\omega_{m m}<0$. On the trailing flank, where the flow direction is opposite to the propagation direction of the wave packet, the Doppler shift of the wave-induced flow on the waves increases the vertical group velocity causing the trailing edge to advance toward the leading edge.

We have performed simulations with $m=-0.7 k$ and $m=-1.4 k$ for which all other wave packet parameters were identical to the previously discussed case. Snapshots from these simulations (not shown) revealed that the tilting of the vertical displacement amplitude envelope was less pronounced in the case with $m=-0.7 k$, and negligible in the simulation with $m=-1.4 k$.

The centerline momentum time series found for a wave packet with $m=-1.4 k$ is shown in Fig. $4 c$. As anticipated, spreading associated with increased vertical group velocity is observed on the leading flank of the wave packet where $U>0$ and $\omega_{m m}>0$. At approximately $t=150 N^{-1}$, the negative wave-induced momentum on the trailing flank begins to narrow and grow in amplitude as the induced flow becomes sufficiently large in magnitude to Doppler-shift the waves to smaller vertical group velocity. At $t \approx 180 N^{-1}$, a positive induced flow develops below the negative flow. This feature arises from the existing positive flow due to wave spreading associated with modulational stability, hence accumulation below the more slowly translating negative flow. A time series of $\|\widetilde{M}\|$, given by (37), for this simulation is shown as the solid curve in Fig. 3c. Being less dispersive than waves for which $m=-0.4 k$, the momentum magnitude does not initially noticeably decrease below unity. At $t \approx 150 N^{-1}$, the momentum magnitude begins increasing, indicating that weakly nonlinear effects have become significant. The vertical line located at $t_{\Delta}=210 N^{-1}$, with $t_{\Delta}$ given by (40), indicates that the predicted time at which weakly nonlinear effects were expected to become significant was somewhat overestimated.

The case of a near-marginally unstable wave packet with $m=-0.7 k$ (for which $\omega_{m m} \lesssim 0$ ), is shown in Fig. 4 b. 
a) $m=-0.4 k$

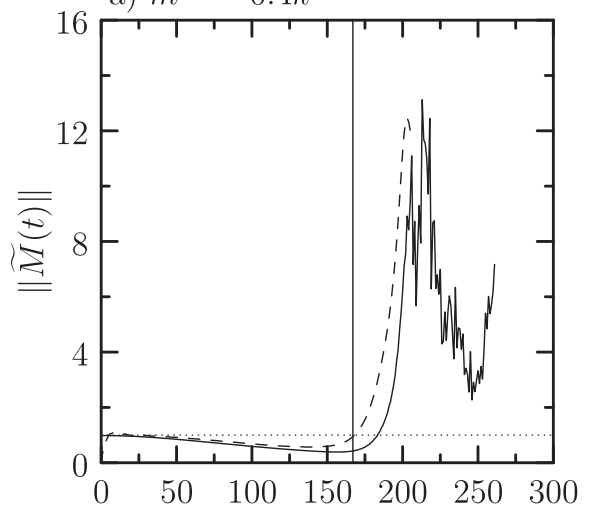

d) $m=-0.4 k$

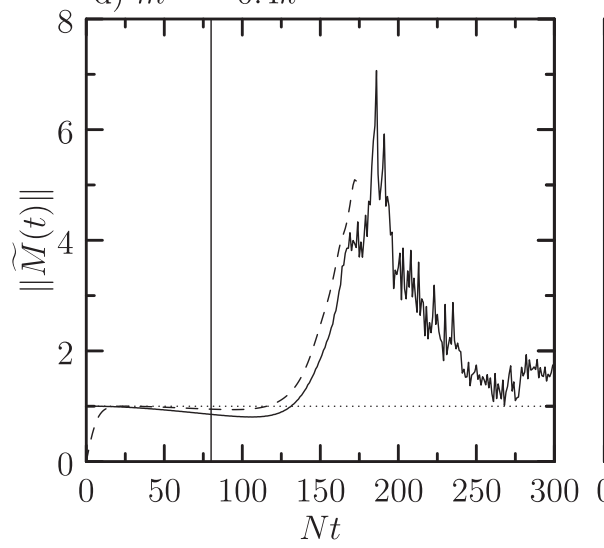

b) $m=-0.7 k$

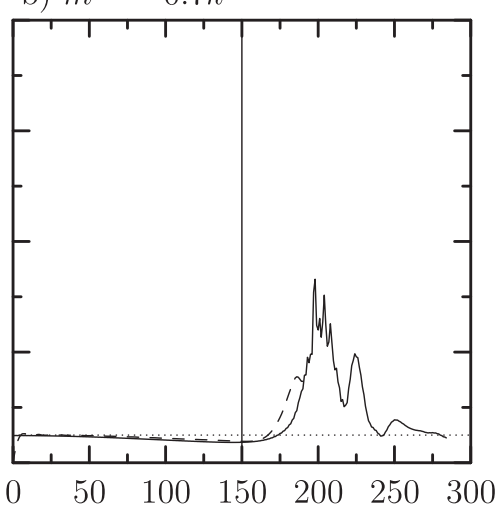

e) $m=-0.7 k$

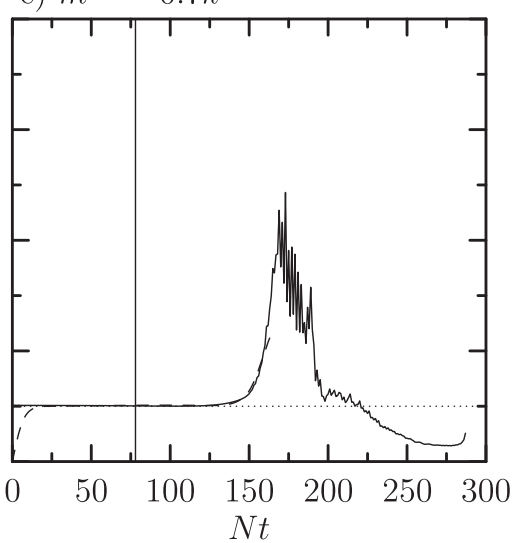

c) $m=-1.4 k$

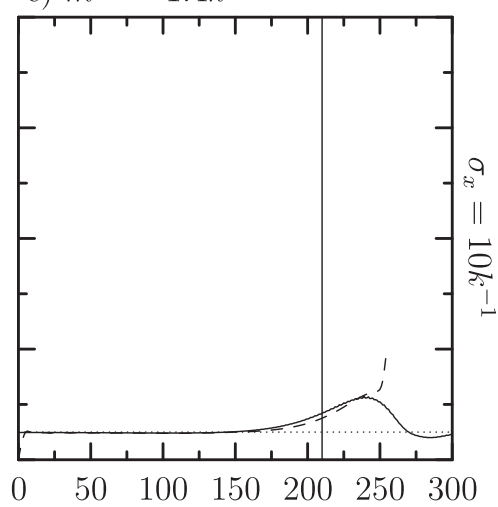

f) $m=-1.4 k$

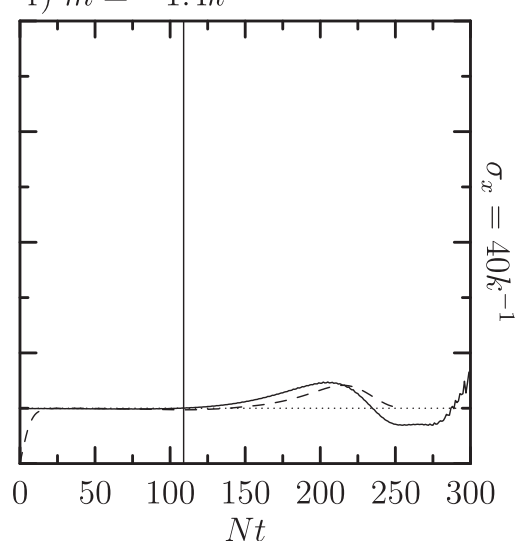

FIG. 3. Time series of the $L^{2}$ norms of relative wave-induced momentum profiles, as given by (37), from the weakly (solid curves) and fully nonlinear (dashed curves) simulations for relative vertical wavenumbers $m$ equal to (a),(d) $-0.4 k$; (b),(e) $-0.7 k$; and (c),(f) $-1.4 k$. All simulations used the constant density scale height $H_{\rho}=10 k^{-1}$. In (a)-(c), the horizontal extent of the wave packet is $\sigma_{x}=10 k^{-1}$, while the simulations in (d)-(f) used $\sigma_{x}=40 k^{-1}$. The solid vertical lines indicate the times $t_{\Delta}$ given by (40), at which weakly nonlinear effects are predicted to become significant.

In this case the dynamics resemble a combination of the previously mentioned cases: there is some spreading across the vertical extent of the wave packet, and at approximately $t=150 N^{-1}$, a negative jet begins to form out of the negative part of the wave-induced mean flow, followed shortly thereafter by a positive jet below it. Almost simultaneously, a series of positive and negative jets begins to form at the leading edge of the wave packet, developing in time toward the center of the wave packet. A time series of $\|\widetilde{M}\|$ for this simulation is shown as the solid curve in Fig. 3b. The observed evolution of the waveinduced momentum magnitude is likewise a combination of the evolution observed in Figs. 3a and 3c: the magnitude decreases due to dispersion, although not to the same degree as in the $m=-0.4 k$ case (see Fig. 3a), and when weakly nonlinear effects become significant, the maximum magnitude is smaller than in the case with $m=-0.4 k$, but greater than in the case with $m=-1.4 k$ (Fig. 3c). Weakly nonlinear effects were predicted to become significant at
$t_{\Delta}=150 N^{-1}$, with $t_{\Delta}$ given by (40), which is somewhat earlier than the observed time, $t \approx 170 N^{-1}$, at which nonlinear effects became significant.

In comparison with the results of DS11 for horizontally periodic wave packets, the times at which weakly nonlinear effects become apparent are consistently later. We have also performed weakly nonlinear simulations for wave packets with longer horizontal extents $\sigma_{x}=40 k^{-1}$. The corresponding time evolution of $\|\widetilde{M}\|$ is shown as the solid curves in Figs. 3d-f. As expected, this shows earlier onset of weakly nonlinear effects. The predicted $t_{\Delta}$ values are in fairly good agreement with the weakly nonlinear simulation in the case with $m=-1.4 k$ and are somewhat underestimated in the cases with $m=-0.4 k$ and $m=-0.7 k$.

\section{b. Fully nonlinear simulations}

Here we compare the results of the previous section with those of fully nonlinear numerical simulations. 

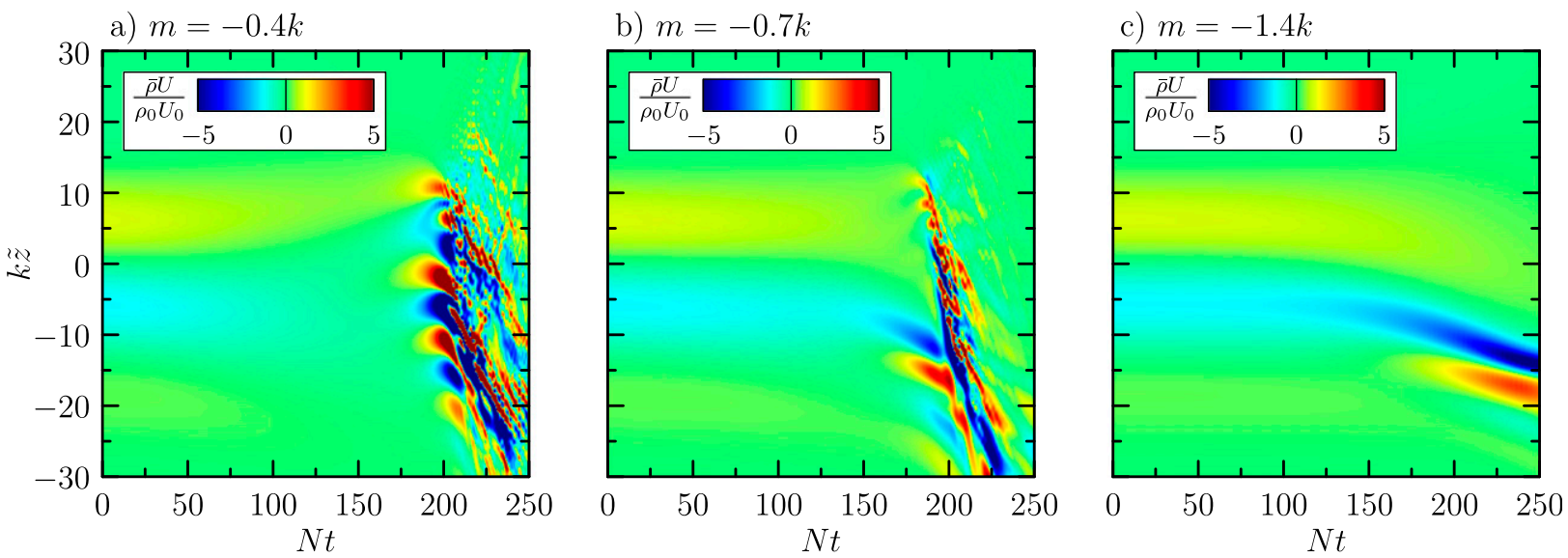

FIG. 4. Time series of density-scaled wave-induced mean-flow profiles $\bar{\rho} U(z, t)$ through the horizontal center of translating wave packets having vertical wavenumbers $m$ equal to (a) $-0.4 k$, (b) $-0.7 k$, and (c) $-1.4 k$, as computed from the weakly nonlinear simulations. Each simulation was initialized with a bivariate Gaussian wave packet according to (30) with initial amplitude $A_{0}=0.05 k^{-1}$ and width $\sigma_{x}=\sigma_{z}=10 k^{-1}$. The waves propagate through an atmosphere with density scale height $H_{\rho}=10 k^{-1}$. In each panel, the wave-induced mean flow is normalized by its peak initial value $\rho_{0} U_{0}$, determined from the numerical output at $t=0$, and plotted in a frame of reference translating at the vertical group velocity $\tilde{z}=z-c_{g_{z}} t$.

Doing so will enable us to assess to what extent weakly nonlinear theory is valid. All fully nonlinear simulations reported upon were initialized with a Gaussian wave packet given by (30) with $A_{0}=0.05 k^{-1}, \sigma_{z}=10 k^{-1}$, and $H_{\rho}=10 k^{-1}$.

First, by comparison with the weakly nonlinear results shown in Fig. 2, the evolution of a wave packet for which $m=-0.4 k$ and $\sigma_{x}=10 k^{-1}$ is examined. At early times, snapshots (not shown) strongly resemble the fields predicted by the weakly nonlinear simulations. For example, the maximum vertical displacements at $t=100 N^{-1}$ in the fully and weakly nonlinear simulations are $\xi=0.255 k^{-1}$ and $\xi=0.249 k^{-1}$, respectively. The similarity among the results at this time suggests that the diffusive effects introduced via the rightmost terms in (33) and (34) contribute insignificantly to the wave packet dynamics. A fully nonlinear simulation initialized with the same parameters, but with doubled values of $C_{\xi}$ and $C_{\zeta}$, revealed no significant qualitative or quantitative differences. Our fully nonlinear results furthermore suggest that dispersion and nonlinearity are captured to a sufficient degree by our nonlinear Schrödinger equation. Qualitative agreement persists at least until wave overturning is first diagnosed at $t=187 N^{-1}$. However, quantitative agreement has deteriorated: the maximum vertical displacements in the fully and weakly nonlinear simulations are $\xi=1.272 k^{-1}$ and $\xi=1.046 k^{-1}$, respectively.

For comparison with the time series constructed from the weakly nonlinear simulations, as shown in Fig. 4, time series constructed from the corresponding fully nonlinear simulations are shown in Fig. 5. In both simulations with $m=-0.4 k$ (Figs. 4a, 5a), the waveinduced mean flow degenerates into a series of positively and negatively flowing jets. Although there are fewer such features in the fully nonlinear simulation, stronger velocities develop earlier on. A time series of $\|\widetilde{M}\|$ computed from the fully nonlinear simulation is shown for the case with $m=-0.4 k$ as the dashed curve in Fig. 3a. That the solid and dashed curves overlap demonstrates excellent agreement among the weakly and fully nonlinear results until $t \approx 150 N^{-1}$, until which time the wave packets exhibit linear evolution. Thereafter, the wave-induced momentum magnitude from the fully nonlinear simulation begins to diverge from that from the weakly nonlinear simulation. Both $L^{2}$ norms increase until just beyond $t=187 N^{-1}$, the first time wave overturning was recorded in the fully nonlinear simulation. The overturning height recorded by the fully nonlinear simulation, $z_{b} \approx 65.9 k^{-1}$, is $\sim 17 \%$ lower than the overturning height predicted by linear theory, $z_{b, L} \approx 78.1 k^{-1}$, with $z_{b, L}$ given by (42). Though overturning, there is a delay before the waves break convectively (Sutherland 2001). In particular, convection takes another three buoyancy periods to develop, after which the code cannot resolve the small and fast convective scales. Having become numerically unstable, the simulation terminates.

The qualitative behavior of waves with $m=-1.4 k$ (Figs. $5 \mathrm{c}$ and $4 \mathrm{c}$, respectively) was captured very well by both the fully and weakly nonlinear simulations until very late times, well beyond the first recorded instance of wave overturning. Approaching the simulation end time, a series of finescale jets develop out of the 

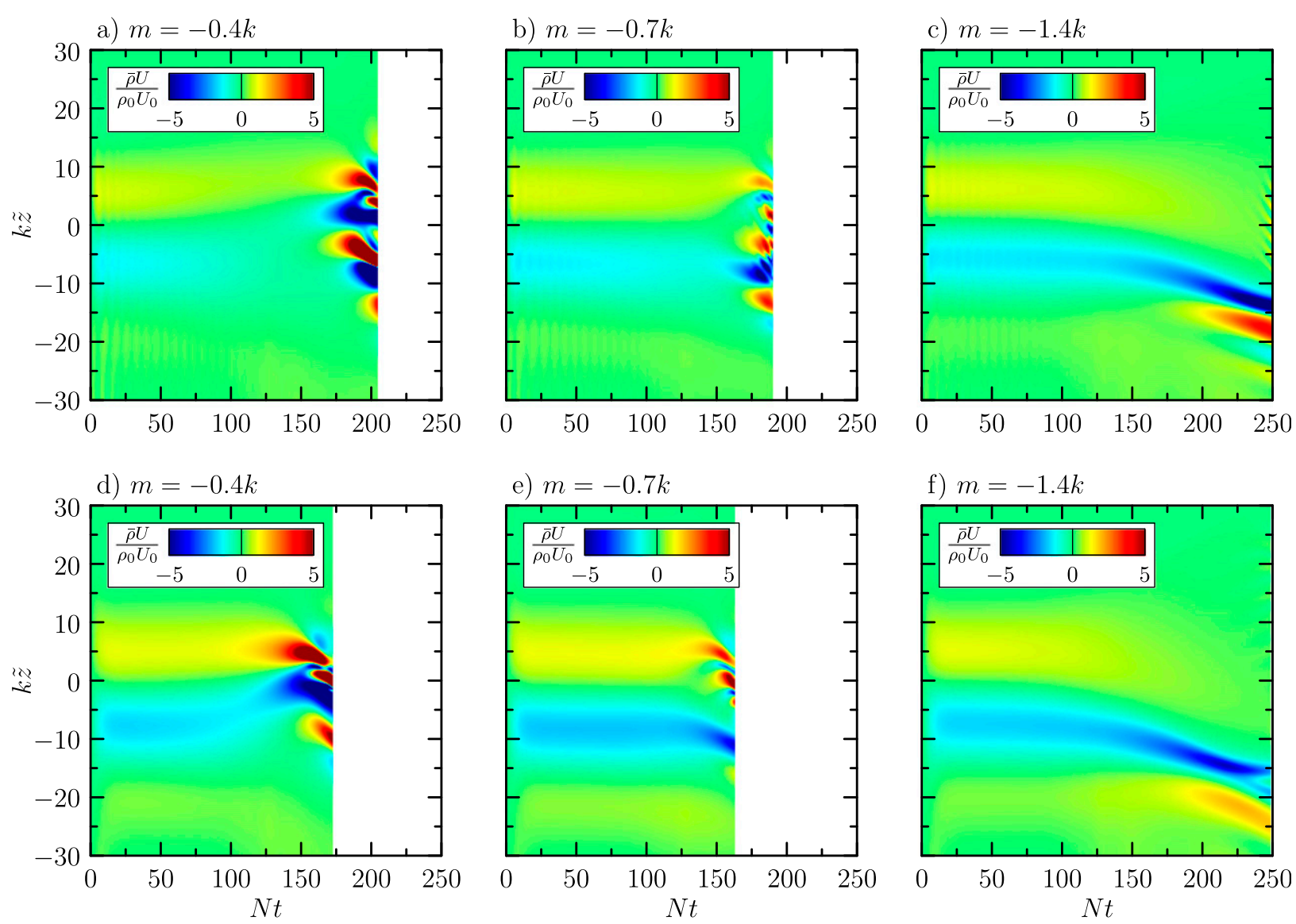

FIG. 5. Time series of density-scaled wave-induced mean-flow profiles $\bar{\rho} U(z, t)$ through the horizontal center of translating wave packets, as computed from fully nonlinear simulations. (top) Each simulation was initialized with a bivariate Gaussian wave packet according to (30) with initial amplitude $A_{0}=0.05 k^{-1}$, width $\sigma_{x}=\sigma_{z}=10 k^{-1}$, and vertical wavenumber $m$ equal to (a) $-0.4 k$, (b) $-0.7 k$, and (c) $-1.4 k$. The waves propagate through an atmosphere with density scale height $H_{\rho}=10 k^{-1}$. (d)-(f) The simulations were initialized with identical parameters as in (a)-(c), but with horizontal wave packet extent $\sigma_{x}=40 k^{-1}$. Each panel is normalized by the peak value of the predicted initial wave-induced mean flow as computed via (B4) and plotted in a frame of reference translating at the vertical group velocity $\tilde{z}=z-c_{g_{z}} t$.

broadened positive part of the wave-induced mean flow that were not resolved by the weakly nonlinear simulation. Before overturning is first observed there is a relative increase in the wave-induced mean flow magnitude. Despite becoming overturning at $t=201 N^{-1}$, the waves continue to evolve for over eight buoyancy periods until the code becomes numerically unstable. A time series of $\|\widetilde{M}\|$ for this simulation is shown as the dashed curve in Fig. 3c. That the solid and dashed curves overlap demonstrates excellent quantitative agreement between the weakly and fully nonlinear results until $t \approx 230 N^{-1}$, that is, through times spanning the wave packet's linear and nonlinear evolution regimes. At very late times in the fully nonlinear results there is a divergence in the $L^{2}$ norm of the centerline wave-induced momentum profile. This is due to the growth of a nonphysical feature near the bottom of the domain. As such we accord this feature no dynamical significance. The height at which overturning was first recorded was $z_{b} \approx 59.7 k^{-1}$, approximately $12 \%$ higher than the height predicted by linear theory, $z_{b, L} \approx 53.2 k^{-1}$.

Fully nonlinear simulations in which the horizontal wave packet extent was quadrupled to $\sigma_{x}=40 k^{-1}$ are shown via time series of their centerline wave-induced momentum profiles in Figs. 5d-f. All other parameters were identical to the corresponding simulations shown in Figs. 5a-c. Qualitatively, the dynamics are similar to their corresponding simulations with $\sigma_{x}=10 \mathrm{k}^{-1}$. The most apparent differences between these results and the previously discussed cases is that the onset of weakly nonlinear effects and the times at which wave overturning is first observed are consistently earlier. Both of these are partly due to the initial magnitude of the waveinduced mean flow-and hence the strength of the nonlinearity in the Schrödinger equation [(28)]—being 
approximately 4 times greater than in the previous cases as predicted by (B4). Long wave packets are also more quasi monochromatic and hence less dispersive, as is evident from the horizontal lines in Figs. 3d-f for early times. Hence, the long wave packets overturn at lower heights than their counterparts with $\sigma_{x}=10 k^{-1}$. In particular, the long wave packets with vertical wavenumbers $m=-0.4 k,-0.7 k$, and $-1.4 k$ overturned at $z_{b} \approx 52.1 k^{-1}, 58.1 k^{-1}$, and $55.8 k^{-1}$, respectively. These values are lower than the overturning heights of their counterparts with $\sigma_{x}=10 k^{-1}$ by approximately $21 \%$, $14 \%$, and $7 \%$, respectively.

Time series of $\|\widetilde{M}\|$ from fully nonlinear simulations in which $\sigma_{x}=40 k^{-1}$ are shown as the dashed lines in Figs. 3d-f, corresponding to relative vertical wavenumbers $m$ equal to $-0.4 k,-0.7 k$, and $-1.4 k$, respectively. For all relative vertical wavenumbers under consideration, both the fully and weakly nonlinear simulations behave in a qualitatively similar way to their counterparts using $\sigma_{x}=10 k^{-1}$, shown respectively in Figs. $3 \mathrm{a}-\mathrm{c}$. In all cases the times $t_{\Delta}$, given by (40), at which weakly nonlinear effects are predicted to become significant are earlier than the predictions for the wave packets with $\sigma_{x}=10 k^{-1}$, as anticipated from (B4). In all cases, the predicted times $t_{\Delta}$ at which weakly nonlinear effects are predicted to become significant appear to be underestimated.

\section{c. Overturning heights}

The fully nonlinear simulations presented in the previous section have demonstrated that wave overturning heights are significantly affected by nonlinear processes. The various cases have shown that a wave packet whose leading flank is prone to narrow and grow in amplitude tends to overturn somewhat below the height predicted by linear theory, while a wave packet whose trailing flank is prone to narrow and grow in amplitude tends to retard linear anelastic growth and overturn slightly above the predicted overturning height.

Here we investigate this further by considering how the density scale height, relative vertical wavenumber, and horizontal wave packet extent affect the location of the overturning height. The overturning heights from fully nonlinear simulations with a range of relative vertical wavenumbers, density scale heights, and horizontal wave packet extents are shown in Fig. 6. Each simulation was initialized with a bivariate Gaussian wave packet as per (30) with amplitude $A_{0}=0.05 k^{-1}$ and vertical wave packet extent $\sigma_{z}=10 k^{-1}$. The horizontal extents $\sigma_{x}$ are equal to either $10 k^{-1}$ (open circles) or $40 k^{-1}$ (crosses). In each panel, the solid curve indicates the breaking height $z_{b, L}$ predicted by linear theory according to (42), and the dashed curve indicates the height $z_{\Delta}$, at which nonlinear effects are predicted to become nonnegligible, given by (39).

As anticipated for waves of relative vertical wavenumber $m=-0.4 k$ (Fig. 6a), modulational instability along the leading flank compounded by linear anelastic growth has caused the wave packet to begin overturning somewhat below the level predicted by linear theory. Wave packets with quadruple horizontal extent overturn at lower height due to the larger wave-induced mean flow and correspondingly earlier onset of weakly nonlinear effects.

Conversely, in the case with $m=-1.4 k$ (Fig. 6c), weakly nonlinear spreading along the leading flank of the wave packet acts to slow anelastic growth. Thus the wave packet first overturns somewhat above the height predicted by linear theory.

In the case with $m=-0.7 k$ (Fig. 6b), overturning begins at almost exactly the height predicted by linear theory for wave packets of horizontal extent $\sigma_{x}=10 \mathrm{k}^{-1}$, and near or below this level for relatively long wave packets.

Figure 6d combines the results shown in Figs. $6 \mathrm{a}-\mathrm{c}$ for fixed density scale height $H_{\rho}=10 \mathrm{k}^{-1}$ and shows that the simulated overturning height is progressively nearer to the breaking height predicted by linear theory as the relative vertical wavenumber increases in absolute value. This is opposite to the results for the horizontally periodic wave packets considered by DS11, who showed that the simulated overturning height becomes progressively higher than the breaking height predicted by linear theory as the relative vertical wavenumber increased in absolute value. Furthermore, Fig. 6d suggests that as wave packets become more hydrostatic, the effect of the horizontal wave packet extent on breaking height becomes less important.

\section{Discussion and conclusions}

As a nontrivial extension of the study of horizontally periodic anelastic internal gravity wave packets by DS11, we have derived the wave-induced mean flow and the nonlinear Schrödinger equation for horizontally and vertically localized wave packets in a uniformly stratified, nonrotating anelastic atmosphere. The nontrivial nonlinear term appropriately captures the interaction of the waves and their induced mean flow, whose analytic form has been derived here for the first time. Comparisons of the numerical solutions of the nonlinear Schrödinger equation with the results of fully nonlinear simulations revealed that the nonlinear Schrödinger equation captures well the weakly nonlinear dynamics of wave packets in this idealized atmosphere. In some cases the nonlinear Schrödinger equation continued to capture 

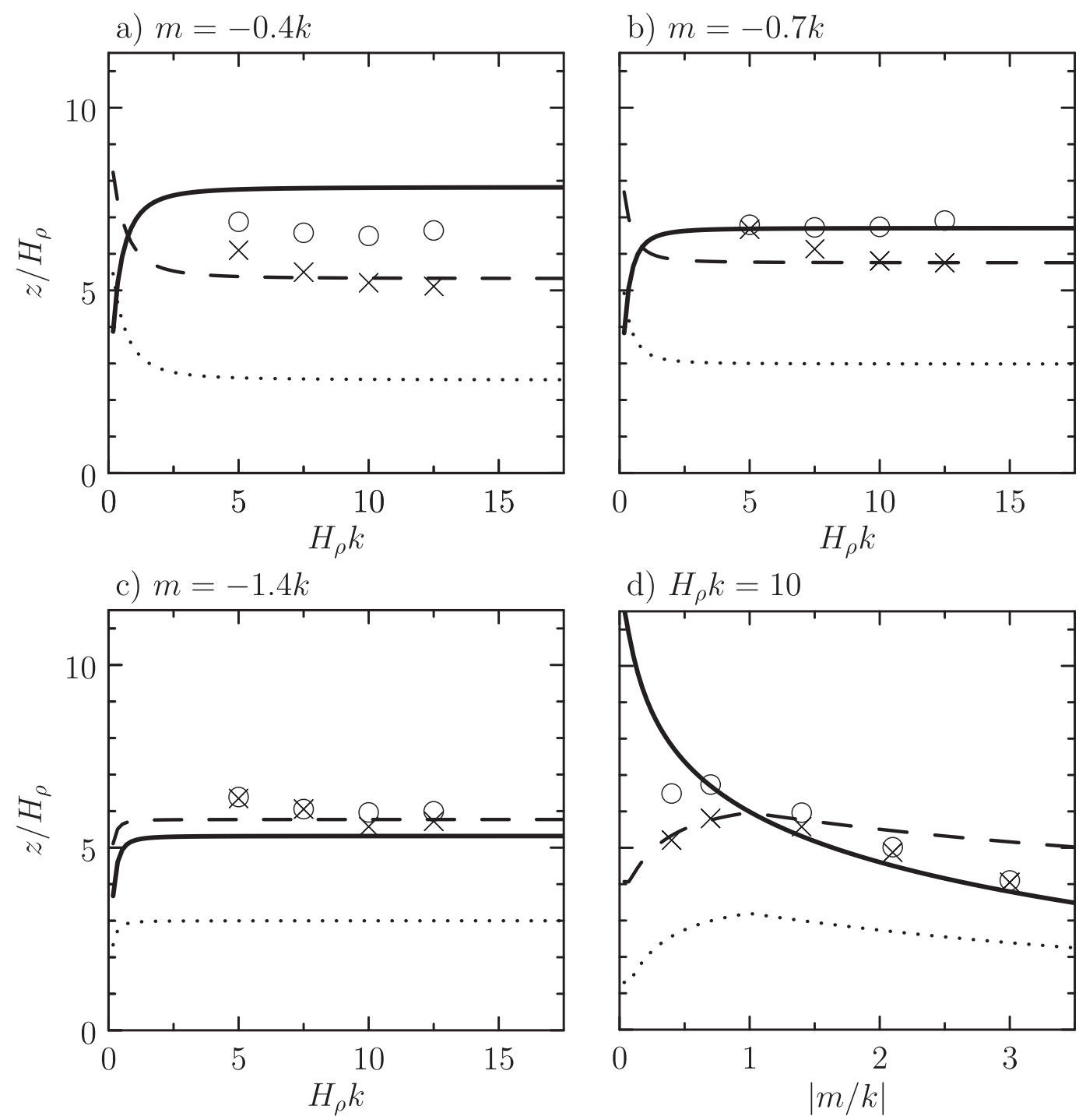

d) $H_{\rho} k=10$

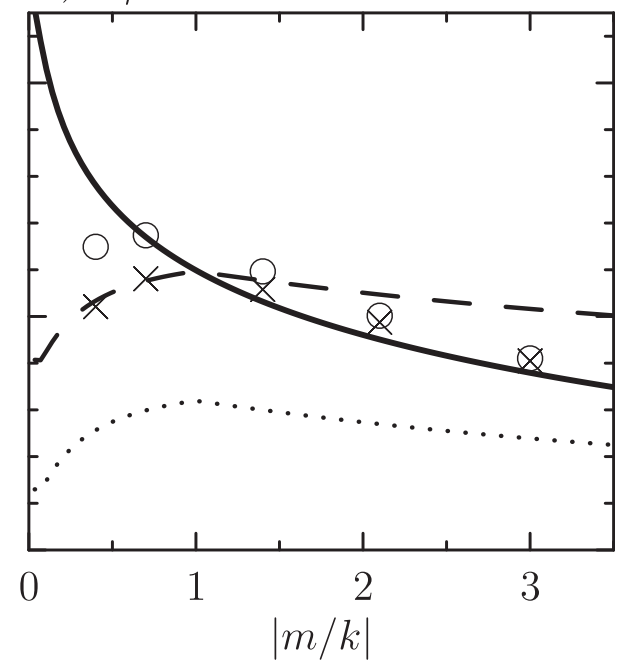

FIG. 6. Heights at which wave packets begin to overturn as computed from fully nonlinear simulations using a range of horizontal wave packet extents, density scale heights, and relative vertical wavenumbers $m$ equal to (a) $-0.4 k$, (b) $-0.7 k$, and (c) $-1.4 k$. (d) The constant density scale height is $H_{\rho} k=10$ and the relative vertical wavenumber is varied. Each simulation was initialized according to (30) with $A_{0}=0.05 k^{-1}$ and $\sigma_{z}=10 k^{-1}$. In each panel, wave packets have either horizontal extent $\sigma_{x}=10 k^{-1}$ (open circles) or $\sigma_{x}=40 k^{-1}$ (crosses). The breaking height predicted by linear theory $z_{b, L}$, given by (42), is indicated by the solid curve; the heights $z_{\Delta}$ at which nonlinear effects are predicted to become nonnegligible for $\sigma_{x}=10 \mathrm{k}^{-1}$ and $\sigma_{x}=40 \mathrm{k}^{-1}$ are indicated by the dashed and dotted curves, respectively, with $z_{\Delta}$ given by (39).

the fully nonlinear dynamics at times beyond the first recorded instance of wave overturning. Our twodimensional equation is suitable for nonhydrostatic waves having relatively narrow or long horizontal extents.

Because the flow induced by a horizontally and vertically localized wave packet changes sign from the leading flank to the trailing flank of the wave packet, twodimensional internal gravity wave packets are modulationally unstable for any relative vertical wavenumber.
A combination of relative vertical wavenumber and the direction of the wave-induced mean flow determines whether narrowing and amplitude growth associated with modulational instability are focused on the leading flank or the trailing flank of the wave packet. In turn this determines whether such waves break somewhat below or just above the height predicted by linear theory.

Qualitative and quantitative similarities among the time series of wave-induced mean-flow profiles, from both weakly and fully nonlinear simulations, show that 
weakly nonlinear theory well captures the early to midlife evolution of internal gravity wave packets as their amplitude grows from relatively small to moderately large. When relative vertical wavenumbers tend toward values corresponding to progressively more hydrostatic waves, qualitative agreement among the weakly and fully nonlinear simulations extends longer into the wave packet's evolution. In one case this agreement persisted well beyond the time at which overturning was first recorded.

For comparison, we revisit the example considered by DS11. We consider a model atmosphere whose density scale height is $H_{\rho}=10 \mathrm{~km}$. Taking $k H_{\rho}=10$ gives a horizontal wavelength of $\lambda_{x} \approx 6 \mathrm{~km}$, and a relative vertical wavenumber of $m / k=-3.0$ corresponds to a wave packet of vertical wavelength $\lambda_{z} \approx 2 \mathrm{~km}$. An initial vertical displacement amplitude of $A_{0}=0.05 \mathrm{k}^{-1}$ corresponds to an actual displacement of $50 \mathrm{~m}$ near the source of wave packet generation. If the wave packet is horizontally periodic, the simulations of DS11 suggest it should begin overturning after propagating upward approximately 11 density scale heights, approximately $190 \%$ higher than the level predicted by linear theory. Conversely, if the wave packet is horizontally localized, our simulations show that overturning should occur after propagating upward only four density scale heights, approximately $5 \%$ higher than the height predicted by linear theory. In our model atmosphere this corresponds to a difference in breaking heights of $\sim 530 \mathrm{~m}$. Alternatively, if the relative vertical wavenumber is $m / k=-0.4$, then the wave packet has vertical wavelength $\lambda_{z} \approx 16 \mathrm{~km}$, and the breaking height predicted by linear theory differs from that resolved by our simulations by $\sim 12.8 \mathrm{~km}$.

Two-dimensional internal gravity wave packets behave in qualitatively different ways than their onedimensional counterparts. Recent studies of fully three-dimensional Boussinesq wave packets (Tabaei and Akylas 2007; van den Bremer and Sutherland 2018) suggest that horizontally, vertically, and spanwise localized wave packets behave differently again. Future work will examine the fully and weakly nonlinear evolution of three-dimensional anelastic internal gravity wave packets with and without the Coriolis force, with the intent ultimately to develop more realistic gravity wave drag parameterization schemes.

Acknowledgments. This research was supported by the Natural Science and Engineering Research Council (NSERC) of Canada Discovery Grant Program. T.S.v.d.B. was partially supported by the Faculty of Science at the University of Alberta through its Visiting Fellowship program.

\section{APPENDIX A}

\section{Explicit Nonlinear Forcing Equation}

Here we follow the approach taken by van den Bremer and Sutherland (2014) to determine the leadingorder nonzero expression for $\bar{\rho} \nabla \cdot \mathbf{F}$ in (12), where the nonlinear vector $\mathbf{F}$ is given by

$$
\mathbf{F} \equiv\left[\partial_{t}\left(\mathbf{u}^{(1)} \xi^{(1)}\right)+N^{2} \partial_{x}\left(\mathbf{u}^{(1)} \xi^{(1)}\right)-\frac{N^{2}}{H_{\rho}}\left(w^{(1)} \xi^{(1)} \hat{\mathbf{e}}_{x}\right)\right],
$$

whose constituent fields are in terms of a quasimonochromatic internal gravity wave packet. Aside from employing the anelastic, rather than Boussinesq, approximation in the present derivation, the chief difference between ours and that of van den Bremer and Sutherland (2014) is that here we do not impose a horizontal Gaussian structure on the wave packet a priori, working instead with a generic amplitude function $\mathcal{A}$ for the sake of generality. We can neglect dispersion, expressed as dependence on the slow variable $T=\varepsilon^{2} t$ in the amplitude envelope functions, so that the leadingorder vertical displacement field is written as

$$
\xi^{(1)}=\xi_{0}^{(1)} \equiv A(X, Z) e^{i \rho+z / 2 H_{\rho}},
$$

where $\varphi \equiv k x+m z-\omega t$ is the phase, and $X=\varepsilon\left(x-c_{g_{x}} t\right)$ and $Z=\varepsilon\left(z-c_{g_{z}} t\right)$ are the slow spatial variables that translate, respectively, at the horizontal and vertical group velocities. As each constituent field of $\mathbf{F}$ is $O(\alpha)$, we will henceforth omit the superscript (1) and employ the perturbation expansion $Q=Q_{0}+\varepsilon Q_{1}+\varepsilon^{2} Q_{2}+\cdots$, where $Q$ denotes any field of interest. To close the set of $O(\alpha \varepsilon)$ polarization relations it is necessary to impose the leading-order vertical displacement field, and we therefore set $\xi_{n} \equiv 0 \forall n \geq 1$.

The remaining fields are obtained by taking derivatives of the amplitude envelope function, since firstorder $X$ and $Z$ derivatives contribute one order in $\varepsilon$. As an example, here we derive the $O(\alpha \varepsilon)$ expression for the mass streamfunction from $w=\partial_{t} \xi=\bar{\rho}^{-1} \partial_{x} \Psi$, which gives

$$
-\left(c_{g_{x}} A_{X}+c_{g_{z}} A_{Z}\right) e^{i \varphi+z / 2 H_{\rho}}=-\frac{\omega}{k} A_{X} e^{i \varphi+z / 2 H_{\rho}}+\frac{1}{\bar{\rho}} i k \Psi_{1},
$$

where the subscripts on $A$ denote partial derivatives. Upon substitution of $c_{g_{x}}=N\left(K^{2}-k^{2}\right) K^{-3}$ and $c_{g_{z}}=-N k m K^{-3}$ (with $K^{2}=k^{2}+m^{2}+1 / 4 H_{\rho}^{2}$ ) into the above equation, we may solve for $\Psi_{1}$, the result of which is

$$
\Psi_{1}=-i \bar{\rho} \frac{N}{K^{3}}\left(k A_{X}+m A_{Z}\right) e^{i \varphi+z / 2 H_{\rho}} .
$$


Using $\Psi \approx \Psi_{0}+\varepsilon \Psi_{1}$ and the $O\left(\alpha \varepsilon^{0}\right)$ polarization relations (included in the center column of Table 1) yields the remaining $O(\alpha \varepsilon)$ fields, given in the rightmost column of Table 1.

Having expressions for each field, we are now able to determine expressions for the slowly varying products of each pair of fields in $\mathbf{F}$. We will explicitly write only the real part of each expression, and omit rapidly varying terms, that is, terms containing the factor $e^{ \pm i 2 \varphi}$. As shown in section 2a, at $O\left(\alpha^{2} \varepsilon^{0}\right)$, we have $\mathbf{F}=0$. The $O\left(\alpha^{2} \varepsilon\right)$ fields are found by multiplying the $O\left(\alpha \varepsilon^{0}\right)$ and $O(\alpha \varepsilon)$ fields and averaging, the results of which are

$(\overline{u \zeta})_{1}^{(2)}=\frac{\varepsilon}{4} \frac{N^{2}}{K^{2}}\left[2 k m \partial_{X}+\left(m^{2}-k^{2}-\frac{1}{4 H_{\rho}^{2}}\right) \partial_{Z}\right]|A|^{2} e^{z / H_{\rho}}$,

$(\overline{w \zeta})_{1}^{(2)}=\frac{\varepsilon}{4} \frac{N^{2}}{K^{2}}\left[\left(m^{2}-k^{2}+\frac{1}{4 H_{\rho}^{2}}\right) \partial_{X}-2 k m \partial_{Z}\right]|A|^{2} e^{z / H_{\rho}}$,

(A6) $(\overline{u \xi})_{1}^{(2)}=\frac{\varepsilon}{4} \frac{N}{K^{3}}\left[-k m \partial_{X}+\left(k^{2}+\frac{1}{4 H_{\rho}^{2}}\right) \partial_{Z}\right]|A|^{2} e^{z / H_{\rho}}$,

$(\overline{w \xi})_{1}^{(2)}=\frac{\varepsilon}{4} \frac{N}{K^{3}}\left[-\left(m^{2}+\frac{1}{4 H_{\rho}^{2}}\right) \partial_{X}+k m \partial_{Z}\right]|A|^{2} e^{z / H_{\rho}}$,

where $|\cdot|$ denotes the modulus. That there are neither $x$ nor $t$ derivatives applied to the rightmost term in $\mathbf{F}$ (as was the case for the first two terms in $\mathbf{F}$ ) suggests the need to include the contribution of $(\overline{w \xi})_{2}^{(2)}$, using the product of the $w_{2}$ and $\xi_{0}$ fields. However, we find that the coefficient on $w_{2}$ is purely imaginary. Hence, because the coefficient on $\xi_{0}$ is purely real, we find that $(\overline{w \xi})_{2}^{(2)} \equiv 0$. Substituting the expressions (A5)-(A8) into $\mathbf{F}$, taking the $t$ and $x$ derivatives, and finally taking the divergence of the resulting expression, yields the $O\left(\alpha^{2} \varepsilon^{3}\right)$ expression for $\nabla \cdot \mathbf{F}$. Explicitly,

$$
(\nabla \cdot \mathbf{F})_{3}^{(2)}=-\frac{\varepsilon^{3}}{4} \frac{N^{3}}{K^{5}} e^{z / H_{\rho}}\left\{\begin{array}{l}
k m\left(3 K^{2}-2 k^{2}\right) \partial_{X X X}+\left[3 m^{4}-4 k^{2} m^{2}-k^{4}+3\left(m^{2}-k^{2}\right) \frac{1}{4 H_{\rho}^{2}}\right] \partial_{X X Z} \\
-k m\left(5 m^{2}-k^{2}+\frac{3}{4 H_{\rho}^{2}}\right) \partial_{X Z Z}+2 k^{2} m^{2} \partial_{Z Z Z} \\
-\frac{1}{\varepsilon H_{\rho}} K^{2}\left(K^{2}-k^{2}\right) \partial_{X X}+\frac{1}{\varepsilon H_{\rho}} k m K^{2} \partial_{X Z}
\end{array}\right\}|A|^{2}
$$

\section{APPENDIX B}

\section{Explicit Integral Expression for the Induced Mean Flow}

Here we describe the integration of Eq. (18) with respect to $\kappa$. We subsequently derive an explicit integral expression for the induced mean flow $u^{(2)}(\tilde{x}, \tilde{z})$. To apply residue theory we define $z \equiv z_{R}+i_{z_{I}}$, a complex-valued analog of $\kappa$, and integrate with respect to this variable. The paths along which the integration is performed are semicircular curves whose arcs encircle the singularities in the complex plane, and whose ends are joined by a line segment along the $\kappa$ axis. To integrate over all real $\kappa$, one allows the radius of the arc to become infinite (e.g., Churchill and Brown 1984). The result of the integration yields an explicit (up to the generality of $\mathcal{A}$ ) integral expression for the induced mass streamfunction in real space,

$$
\Psi^{(2)}=\frac{\pi}{2} \rho_{0} \frac{N^{2} k^{2} m^{2}}{\left|c_{g_{z}}\right| K^{5}} A_{0}^{2} \int_{0}^{\infty} I(\mu ; \tilde{x}, \tilde{z}) d \mu
$$

in which the integrand is given by

$$
I(\mu ; \tilde{x}, \tilde{z})=\frac{\mu e^{C_{I}|\tilde{x}|}\left[e^{-i\left(\mu \tilde{z}-C_{R}|\tilde{x}|-\Phi\right)}\left|\widehat{\left.\mathcal{A}\right|^{2}}\right|_{z=C^{\star}(\mu)}+e^{i\left(\mu \tilde{z}-C_{R}|\tilde{x}|-\Phi\right)}\left|\widehat{\left.\mathcal{A}\right|^{2}}\right|_{z=-C(\mu)}\right]}{\left(1+\frac{1}{H_{\rho}^{2} \mu^{2}}\right)^{1 / 4}} .
$$


Here the star denotes the complex conjugate and the subscripts on the $|\widehat{\mathcal{A}}|^{2}$ terms indicate that the functional dependence of $|\widehat{\mathcal{A}}|^{2}$ on $z$ has been replaced by a dependence on $C^{\star}(\mu)$ or $-C(\mu)$, with $C(\mu)=C_{R}+i C_{I}$ defined in (19). The expression for the horizontal flow associated with the induced long wave is readily computed via the relation

$$
u^{(2)}(\tilde{x}, \tilde{z})=-\frac{1}{\bar{\rho}} \partial_{\tilde{z}} \Psi^{(2)}(\tilde{x}, \tilde{z})
$$

Finally, the horizontal component of the local waveinduced mean flow is found by taking a vertical profile of the induced flow through the center of the translating wave packet, that is, by setting $\tilde{x}=0$ on the left-hand side of (B3), which we denote $U(z, t)$.

As an example, for a bivariate Gaussian wave packet, given by $\mathcal{A}(\tilde{x}, \tilde{z})=e^{-\left(\tilde{x}^{2} / 2 \sigma_{x}^{2}+\tilde{z}^{2} / 2 \sigma_{z}^{2}\right)}$, we have from (B1) that

$$
\begin{aligned}
u^{(2)}(\tilde{x}, \tilde{z}) & =-\frac{1}{\bar{\rho}} \partial_{\tilde{z}} \Psi^{(2)}(\tilde{x}, \tilde{z}) \\
& =\frac{1}{4} \frac{N^{2} k^{2} m^{2}}{\left|c_{g_{z}}\right| K^{5}} A_{0}^{2} \sigma_{x} \sigma_{z} e^{z / H_{\rho}} \int_{0}^{\infty} \frac{\partial I}{\partial \tilde{z}} d \mu,
\end{aligned}
$$

where the integrand is given by

$$
\frac{\partial I}{\partial \tilde{z}}(\mu ; \tilde{x}, \tilde{z})=\frac{\mu^{2} e^{\left[C_{I}|\tilde{x}|-\mu^{2} \sigma_{z}^{2} / 4-\left(C_{R}^{2}-C_{I}^{2}\right) \sigma_{x}^{2} / 4\right]} \sin \left(\mu \tilde{z}-C_{R}|\tilde{x}|-C_{R} C_{I} \sigma_{x}^{2} / 2-\Phi\right)}{\left(1+\frac{1}{H_{\rho}^{2} \mu^{2}}\right)^{1 / 4}}
$$

The branch cut corresponding to the outward- and downward-propagating waves we wish to capture is that for which the plus-or-minus sign of $\Phi=$ $\pm(1 / 2) \tan ^{-1}\left(1 / H_{\rho}|\mu|\right)$ is equal to $\operatorname{sgn}(\mu)$, as was done in section 2a. To implement this branch cut, it is also necessary to set $-C_{R}|\tilde{x}| \rightarrow C_{R}|\tilde{x}|$ in (B5). In agreement with the findings of previous studies of Boussinesq wave packets (Bretherton 1969; Tabaei and Akylas 2007), the resulting expression for the induced flow field, $u^{(2)}(\tilde{x}, \tilde{z})$, is not uniformly valid. This fact is inconsequential for our study because we only require profiles of the induced flow through $\tilde{x}=0$, and because we use (B4) for theoretical purposes only.

For illustrative purposes, the initial two-dimensional horizontal flow field induced by the waves, $\left.u^{(2)}(x, z)\right|_{t=0}$, is shown in Fig. 1a. This field was computed in Fourier space using the method described in section 2a. The selection shown was cropped from the actual spatial domain, $|k x| \leq 1608.6$ and $-250 \leq k z \leq 150$. Consistent with previous results (Bretherton 1969; Tabaei and Akylas 2007; van den Bremer and Sutherland 2014), the induced flow field qualitatively resembles a bow wake. In Fig. 1b, the solid curve is the vertical profile of the of the horizontal component of the wave-induced mean flow $U(z, t=0)=\left.u^{(2)}(x=0, z)\right|_{t=0}$. The dashed red curve is computed from the integral expression given by (B4).

Finally, we remark that although (B1) is valid for all time preceding wave overturning, (B4) is valid only at time $t=0$. This is because for any $t>0$ weakly nonlinear effects have begun to modify the wave packet envelope to some degree, and so it is no longer described by its initial Gaussian structure. Because of the impracticality of numerically evaluating a function along a curve, as in terms of the form $\left|\overline{\left.\mathcal{A}\right|^{2}}\right|_{z}=Q(\mu)$, in practice we determine $u^{(2)}$ in Fourier space from (18).

\section{REFERENCES}

Achatz, U., R. Klein, and F. Senf, 2010: Gravity waves, scale asymptotics and the pseudo-incompressible equations. J. Fluid Mech., 663, 120-147, https://doi.org/10.1017/ S0022112010003411.

Acheson, D. J., 1976: On over-reflexion. J. Fluid Mech., 77, 433472, https://doi.org/10.1017/S0022112076002206.

Akylas, T. R., and A. Tabaei, 2005: Resonant self-acceleration and instability of nonlinear internal gravity wavetrains. Frontiers of Nonlinear Physics, A. Litvak, Ed., Institute of Applied Physics, 129-135.

Bretherton, F. P., 1969: On the mean motion induced by gravity waves. J. Fluid Mech., 36, 785-803, https://doi.org/10.1017/ S0022112069001984.

Churchill, R. V., and J. W. Brown, 1984: Complex Variables and Applications. 4th ed., McGraw-Hill, 339 pp.

Dosser, H. V., and B. R. Sutherland, 2011: Anelastic internal wavepacket evolution and stability. J. Atmos. Sci., 68, 28442859, https://doi.org/10.1175/JAS-D-11-097.1.

Eliassen, A., and E. Palm, 1961: On the transfer of energy in stationary mountain waves. Geofys. Publ., 22, 1-23.

Grimshaw, R. H. J., 1981: The modulation of a weakly nonlinear wave packet in an inhomogeneous medium. SIAM J. Appl. Math., 40, 10-34, https://doi.org/10.1137/0140002.

Lindzen, R. S., 1981: Turbulence and stress owing to gravity wave and tidal breakdown. J. Geophys. Res., 86, 9707-9714, https:// doi.org/10.1029/JC086iC10p09707.

Lipps, F. B., and R. S. Hemler, 1982: A scale analysis of deep moist convection and some related numerical calculations. J. Atmos. Sci., 39, 2192-2210, https://doi.org/10.1175/1520-0469(1982) $039<2192$ :ASAODM $>2.0 . \mathrm{CO} ; 2$.

McFarlane, N. A., 1987: The effect of orographically excited gravity wave drag on the general circulation of the lower 
stratosphere and troposphere. J. Atmos. Sci., 44, 1775-1800, https://doi.org/10.1175/1520-0469(1987)044<1775:TEOOEG> 2.0.CO;2.

Ogura, Y., and N. A. Phillips, 1962: Scale analysis of deep and shallow convection in the atmosphere. J. Atmos. Sci., 19, 173-179, https://doi.org/10.1175/1520-0469(1962)019<0173: SAODAS $>2.0 . C O ; 2$.

Palmer, T. N., G. J. Shutts, and R. Swinbank, 1986: Alleviation of a systematic westerly bias in general circulation and numerical weather prediction models through an orographic gravity wave drag parametrization. Quart. J. Roy. Meteor. Soc., 112, 1001-1039, https://doi.org/10.1002/qj.49711247406.

Scinocca, J. F., and T. G. Shepherd, 1992: Nonlinear waveactivity conservation laws and Hamiltonian structure for the two-dimensional anelastic equations. J. Atmos. Sci., 49, 5-27, https://doi.org/10.1175/1520-0469(1992)049<0005: NWACLA $>2.0 . \mathrm{CO} ; 2$.

— , and N. A. McFarlane, 2000: The parameterization of drag induced by stratified flow of anisotropic orography. Quart. J. Roy. Meteor. Soc., 126, 2353-2393, https://doi.org/10.1002/qj.49712656802.

Shrira, V. I., 1981: On the propagation of a three-dimensional packet of weakly non-linear internal gravity waves. Int. J. Nonlinear Mech., 16, 129-138, https://doi.org/10.1016/0020-7462(81) 90004-4.
Sutherland, B. R., 2001: Finite-amplitude internal wavepacket dispersion and breaking. J. Fluid Mech., 429, 343-380, https:// doi.org/10.1017/S0022112000002846.

_ 2006a: Internal wave instability: Wave-wave vs wave-induced mean flow interactions. Phys. Fluids., 18, 074107, https:// doi.org/10.1063/1.2219102.

_ 2006b: Weakly nonlinear internal gravity wavepackets. J. Fluid Mech., 569, 249-258, https://doi.org/10.1017/ S0022112006003016.

_ 2010: Internal Gravity Waves. Cambridge University Press, $378 \mathrm{pp}$.

Tabaei, A., and T. R. Akylas, 2007: Resonant long-short wave interactions in an unbounded rotating stratified fluid. Stud. Appl. Math., 119, 271-296, https://doi.org/10.1111/j.14679590.2007.00389.x.

van den Bremer, T. S., and B. R. Sutherland, 2014: The mean flow and long waves induced by two-dimensional internal gravity wavepackets. Phys. Fluids, 26, 106601, https://doi.org/10.1063/ 1.4899262.

— , and — 2018: The wave-induced flow of wide threedimensional internal gravity wavepackets. J. Fluid Mech., 834, 385-408, https://doi.org/10.1017/jfm.2017.745.

Whitham, G. B., 1974: Linear and Nonlinear Waves. John Wiley and Sons, Inc., 636 pp. 